\title{
Geometrothermodynamics of five dimensional black holes in Einstein-Gauss-Bonnet theory
}

\author{
Safia Taj ${ }^{1,3}$, Hernando Quevedo ${ }^{2,3}$ and Alberto Sánchez ${ }^{4 *}$ \\ ${ }^{1}$ Center for Advanced Mathematics and Physics, \\ National University of Sciences and Technology, H-12, Islamabad, Pakistan \\ ${ }^{2}$ Instituto de Ciencias Nucleares, Universidad Nacional Autónoma de México, \\ AP 70543, México, DF 04510, Mexico \\ ${ }^{3}$ Dipartimento di Fisica and ICRA, \\ Università di Roma La Sapienza, I-00185 Roma, Italy \\ ${ }^{4}$ Departamento de Posgrado, CIIDET, \\ AP 752, Querétaro, QRO 76000, Mexico
}

(Dated: June 8, 2022)

\begin{abstract}
We investigate the thermodynamic properties of 5D static and spherically symmetric black holes in (i) Einstein-Maxwell-Gauss-Bonnet theory, (ii) Einstein-Maxwell-Gauss-Bonnet theory with negative cosmological constant, and in (iii) Einstein-Yang-Mills-Gauss-Bonnet theory. To formulate the thermodynamics of these black holes we use the Bekenstein-Hawking entropy relation and, alternatively, a modified entropy formula which follows from the first law of thermodynamics of black holes. The results of both approaches are not equivalent. Using the formalism of geometrothermodynamics, we introduce in the manifold of equilibrium states a Legendre invariant metric for each black hole and for each thermodynamic approach, and show that the thermodynamic curvature diverges at those points where the temperature vanishes and the heat capacity diverges.
\end{abstract}

PACS numbers: 04.25.Nx; 04.80.Cc; 04.50.Kd

Keywords: Geometrothermodynamics, Einstein-Gauss-Bonnet theory, Phase transition

*Electronic address: safiataaj@gmail.com, quevedo@nucleares.unam.mx 


\section{INTRODUCTION}

Black holes can be regarded as thermodynamical systems [1, 2] which radiate Hawking thermal radiation of temperature proportional to the surface gravity on the horizon of the black hole, and Bekenstein-Hawking entropy proportional to its horizon area [3], 4]. Indeed, these quantities satisfy the first law of black hole thermodynamics [5]. Finding the microscopic description of this entropy is one of the most challenging questions in theoretical physics. The solution to this problem still remains obscure. According to the no-hair theorems of Einstein-Maxwell theory, electro-vacuum black holes are completely described by three parameters only: mass $M$, angular momentum $J$, and electric charge $Q$. All these parameters define the heat capacity whose sign, in turn, determines the thermodynamical stability of the black hole.

During the last few decades several attempts have been made in order to introduce Riemannian geometric concepts in ordinary thermodynamics. First, Weinhold [6] introduced on the space of equilibrium states a metric whose components are given as the Hessian of the internal thermodynamic energy. Later, Ruppeiner [7] introduced a metric which is defined as minus the Hessian of the entropy, and is conformally equivalent to Weinhold's metric, with the inverse of the temperature as the conformal factor. The physical meaning of Ruppeiner's metric lays in the fluctuation theory of equilibrium thermodynamics. It turns out that the second moments of fluctuation are related to the components of Ruppeiner's metric.

One of the aims of the application of geometry in thermodynamics is to describe phase transitions in terms of curvature singularities so that the curvature can be interpreted as a measure of thermodynamic interaction. The study of the relation between the phase space and the metric structures of the space of equilibrium states led to the result that Weinhold's and Ruppeiner's thermodynamic metrics are not invariant under Legendre transformations [8]. On the other hand, ordinary thermodynamics is invariant with respect to Legendre transformations, i. e., the physical properties of a thermodynamic system do not change when different thermodynamic potentials are used [9]. One might then wonder whether the use of non Legendre invariant metric structures in ordinary thermodynamics would always lead to results that do not depend on the thermodynamic potential. Indeed, several examples are known in the literature in which a change of thermodynamic potential leads to a modification of the thermodynamic geometry [10]. Some puzzling results arise also in 
connection with the use of different metrics in the equilibrium space [11], in the sense that for the same thermodynamic system the resulting geometry can be either flat or curved, depending on the chosen metric. Recently, in the analysis of Einstein-Gauss-Bonnet (EGB) black holes inconsistencies were also found [12]. In this work, we will focus on the study of EGB black holes and will clarify these inconsistencies.

Recently, the formalism of geometrothermodynamics (GTD) was developed in order to unify in a consistent manner the geometric properties of the phase space and the space of equilibrium states [13]. Legendre invariance plays an important role in this formalism. In particular, it allows us to derive Legendre invariant metrics for the space of equilibrium states. The thermodynamic phase space $\mathcal{T}$ is assumed to be coordinatized by the set of independent coordinates $\Phi, E^{a}, I^{a}, a=1, \ldots, n$, where $\Phi$ represents the thermodynamic potential, and $E^{a}$ and $I^{a}$ are the extensive and intensive thermodynamic variables, respectively The positive integer $n$ indicates the number of thermodynamic degrees of freedom of the black hole configuration. Moreover, the phase space is endowed with the Gibbs one-form $\Theta=d \Phi-\delta_{a b} I^{a} d E^{b}$, with $\delta_{a b}=\operatorname{diag}(1, \ldots, 1)$, satisfying the condition $\Theta \wedge(d \Theta)^{n} \neq 0$. Consider also on $\mathcal{T}$ a Riemannian metric $G$ which must be invariant with respect to Legendre transformations of the form $\left(\Phi, E^{a}, I^{a}\right) \rightarrow\left(\tilde{\Phi}, \tilde{E^{a}}, \tilde{I^{a}}\right)$, with $\Phi=\tilde{\Phi}-\delta_{a b} \tilde{E}^{a} \tilde{I}^{b}, E^{a}=-\tilde{I^{a}}$, $I^{a}=\tilde{E}^{a}[14]$. We impose the Legendre invariance condition in order to incorporate in GTD the invariance of ordinary thermodynamics with respect to changes of the thermodynamic potential [9]. It turns out that the condition of Legendre invariance is not sufficient to fix uniquely the metric $G$. In fact, Legendre invariance generates the metric

$$
G=\left(d \Phi-I_{a} d E^{a}\right)^{2}+\Lambda\left(\xi_{a b} E^{a} I^{b}\right)\left(\chi_{c d} d E^{c} d I^{d}\right)
$$

where $\Lambda$ is an arbitrary real constant, and $\xi_{a b}$ and $\chi_{a b}$ are diagonal constant tensors. Clearly, the diagonal components of these tensors can be normalized by rescaling the coordinates $E^{a}$ and $I^{a}$, and by choosing the multiplicative constant $\Lambda$ appropriately. Then, one can express $\xi_{a b}$ and $\chi_{a b}$ in terms of the usual Euclidean metric, $\delta_{a b}=\operatorname{diag}(1, \ldots, 1)$, and the pseudoEuclidean metric, $\eta_{a b}=\operatorname{diag}(-1,1, \ldots, 1)$. Physical conditions must be invoked to fix the final form of these tensors [15]. Indeed, it turns out that the choice $\xi_{a b}=\delta_{a b}$ and $\chi_{a b}=\delta_{a b}$ $\left(\xi_{a b}=\delta_{a b}\right.$ and $\left.\chi_{a b}=\eta_{a b}\right)$ leads to a metric which describes systems characterized by first (second) order phase transitions. Moreover, the choice $\xi_{a b}=\left(\delta_{a b}-\eta_{a b}\right) / 2$ allows us also to correctly handle the zero-temperature limit in a geometric way. We see that Legendre 
invariance leaves free only the signature of $\chi_{a b}$. The signature, in turn, is fixed by the order of the phase transition under consideration [50]. Since in this work we will analyze second order phase transitions of EGB black holes [12], we choose the metric as

$$
G=\left(d \Phi-\delta_{a b} I^{a} d E^{b}\right)^{2}+\frac{1}{2}\left(\delta_{a b}-\eta_{a b}\right) E^{a} I^{b}\left(\eta_{c d} d E^{c} d I^{d}\right) .
$$

The set $(\mathcal{T}, \Theta, G)$ defines a Legendre invariant manifold with a contact Riemannian structure. The equilibrium space $\mathcal{E} \subset T$ is defined by the map $\varphi: \mathcal{E} \rightarrow \mathcal{T}$ or, in local coordinates, $\varphi:\left(E^{a}\right) \mapsto\left(\Phi, E^{a}, I^{a}\right)$, satisfying the condition $\varphi^{*}(\Theta)=0$, i. e., on $\mathcal{E}$ it holds the first law of thermodynamics, $d \Phi=\delta_{a b} I^{a} d E^{b}$, and the conditions of thermodynamic equilibrium $I^{a}=\delta^{a b} \partial \Phi / \partial E^{b}$, which relate the extensive variables $E^{a}$ with the intensive ones $I^{a}$. Then, the pullback $\varphi^{*}$ induces on $\mathcal{E}$, by means of $g=\varphi^{*}(G)$, the thermodynamic metric

$$
g=\frac{1}{2}\left[E^{a}\left(\frac{\partial \Phi}{\partial E^{a}}-\eta_{a b} \delta^{b c} \frac{\partial \Phi}{\partial E^{c}}\right)\right]\left(\eta_{a b} \delta^{b c} \frac{\partial^{2} \Phi}{\partial E^{c} \partial E^{d}} d E^{a} d E^{d}\right) .
$$

For the construction of this thermodynamic metric it is only necessary to know explicitly the thermodynamic potential in terms of the extensive variables $\Phi=\Phi\left(E^{a}\right)$. It is worth mentioning that GTD allows us to implement easily different thermodynamic representations [17-22].

Since a thermodynamic system is uniquely determined by the fundamental equation $\Phi=\Phi\left(E^{a}\right)[9]$, the equilibrium space contains the information about the thermodynamic geometry of the system. In this connection, two geometric concepts are very important: the distance and the curvature of $\mathcal{E}$. The metric $g$ determines the thermodynamic distance $d s^{2}=g_{a b} d E^{a} d E^{b}$ along the curve which connects two equilibrium states $E^{a}$ and $E^{a}+d E^{a}$, i. e., two points of the equilibrium manifold $\mathcal{E}$ [13]. As has been shown in [16], the curves satisfying the variational principle $\delta \int d s=0$ and the entropy condition determine thermodynamic geodesics, i. e., extremal curves which describe quasi-static thermodynamic processes.

Notice that in general the signature of $g_{a b}$ is not fixed and so the equilibrium manifold can be either Riemannian or pseudo-Riemannian. This implies that the thermodynamic distance $d s^{2}$ can be either positive, negative or zero. As a consequence, one can show [25, 26] that there exists a casual structure in $\mathcal{E}$ that permits to connect thermodynamically one state with another state, but forbids the connection with some other states. The boundary of causality is determined by the states satisfying the condition $d s^{2}=0$, i. e., states that can be connected by a reversible process. 
Recently, it was pointed out in [23] that the thermodynamic metric $g_{a b}$ can be used to define in the equilibrium manifold $\mathcal{E}$ the probability $P\left(E^{a}\right)$ to find a system within the interval $E^{a}+d E^{a}$ as

$$
P\left(E^{a}\right)=\frac{\sqrt{\left|\operatorname{det}\left(g_{a b}\right)\right|}}{(2 \pi)^{n / 2}} \exp \left(\frac{1}{2} g_{a b} d E^{a} d E^{b}\right) .
$$

Then, $g^{a b}$ turns out to be related to the second moments of fluctuation, in a way that resembles the physical interpretation of Ruppeiner's metric in fluctuation theory. This opens the possibility to relate the thermodynamic curvature of $\mathcal{E}$ with the correlation length of the thermodynamic system described by $\mathcal{E}$. In this case, the determinant and the principal minors of $g_{a b}$ must be positive definite, a property that could be related to the causal structure of $\mathcal{E}$ mentioned above. A more detailed analysis will be necessary to clarify this point.

Moreover, the curvature of $\mathcal{E}$ is interpreted as a measure of the thermodynamic interaction of the system with curvature singularities at those points where phase transitions take place [24]. This interpretation resembles the role of curvature in gravity and gauge theories, i. e., the curvature is a measure of the field interaction with curvature singularities indicating the break down of the theory.

The above interpretation of the thermodynamic curvature in GTD has been proved in several ordinary thermodynamic systems and also in the context of black holes. In the case of the ideal gas, the curvature vanishes and the thermodynamic geodesics are straight lines. This is in accordance with our physical expectations since the ideal gas has no internal thermodynamic interaction. In the case of the van der Waals gas, which has a non-trivial thermodynamic interaction [9], the equilibrium space is curved and the curvature singularities correspond to the points where phase transitions take place. The corresponding thermodynamic geodesics are curved and represent quasi-static processes which, under certain initial conditions, end at those points where phase transitions occur [27], in agreement with the well-known relation between geodesic incompleteness and curvature singularities. For other ordinary thermodynamic systems, like several generalizations of the ideal gas and the Ising model, we obtained similar results. In the context of black holes [15], the results are compatible with those obtained in ordinary black hole thermodynamics, i. e., black holes with thermodynamic interaction possess a curved equilibrium space with singularities at those places where phase transitions occur. In the present work, the correctness of this 
interpretation will be shown for black holes in a particular set of higher dimensional gravity theories.

Since a system with thermodynamic interaction can be either stable or unstable, we expect that the curvature of $\mathcal{E}$ reproduces this behavior and so it can be different from zero, regardless of the stability properties of the system. In fact, we have found so far in GTD only two systems with a flat curvature, namely, the ideal gas [13, 25] and a particular topological black hole with flat horizon in Hořava-Lifshitz gravity [28]. Both cases correspond to stable systems. In some sense, this can be expected intuitively since in a stable system with vanishing curvature there is no thermodynamic interaction that could drive the evolution into another state. On the contrary, an unstable system must naturally evolve into a different state, a process that demands a non-zero thermodynamic interaction and, consequently, a non-flat curvature. In all the thermodynamic systems we have analyzed in GTD so far, an unstable system is always characterized by a non-zero curvature. We will show in this work that this is also true in the case of Einstein-Gauss-Bonnet black holes.

In five dimensions, the most general theory leading to second order field equations for the metric is the so-called Einstein-Gauss-Bonnet (EGB) theory, which contains quadratic powers of the curvature. The most general action of the EGB theory is obtained by adding the Gauss-Bonnet (GB) invariant and the matter Lagrangian $L_{\text {matter }}$ to the Einstein-Hilbert action

$$
I=\kappa \int d^{5} x \sqrt{g}\left[R+\alpha\left(R^{2}-4 R^{\mu \nu} R_{\mu \nu}+R^{\alpha \beta \gamma \delta} R_{\alpha \beta \gamma \delta}\right)+L_{\text {matter }}\right],
$$

where $\kappa$ is related to the Newton constant, and $\alpha$ is the Gauss-Bonnet coupling constant. GB extensions of General Relativity have been motivated from a string theoretical point of view as a version of higher-dimensional gravity, since this sort of modification also appears in low energy effective actions of string theory. The pioneering work in this regard belongs to Boulware and Deser [29]. They obtained the most general static black hole solutions in EGB theory. The GB term has some remarkable features. For instance, in higher dimensions, it is the most general quadratic correction which preserves the property that the equations of motion involve only second order derivatives of the metric [30]. However, in 4D, the GB term is topological in nature and it does not enter the dynamics [31]. The GB term is important from both physical and geometrical points of view; it naturally arises as the next leading order of the $\alpha$-expansion of the heterotic superstring theory ( $\alpha^{-1}$ is the string tension) [32], and plays a fundamental role in Chern-Simons gravitational theories [33]. Several aspects 
of such extended gravity theories have been extensively studied in [34].

In this work, we study the thermodynamics of static spherically symmetric black holes in five dimensional Einstein-Maxwell-Gauss-Bonnet (EMGB) with and without cosmological constant, and in the Einstein-Yang-Mills-Gauss-Bonnet (EYMGB) theory. We use two different approaches to formulate black holes thermodynamics. The first one is based upon the use of the Bekenstein-Hawking entropy relation, and the second one uses a different formula for the entropy which follows from the first law of black hole thermodynamics. We will see that the two approaches lead to completely different thermodynamics that have effects on the stability properties and the phase transition structure of black holes. The approach of GTD is used to show that there exists a Legendre invariant thermodynamic metric for the equilibrium space which in all the cases considered here describes correctly the thermodynamic interaction in terms of the curvature of the equilibrium manifold, and the phase transitions and the point with zero temperature in terms of curvature singularities.

This paper is organized as follows. Section II deals with the thermodynamics and GTD of a particular asymptotically de Sitter black hole solution [35] of EMGB theory. Section [II] is dedicated to the study of an asymptotically anti de Sitter black hole solution of EMGB theory with cosmological constant. A black hole spacetime of EYMGB theory is investigated in Section IV. Finally, Section $\mathrm{V}$ is devoted to conclusions and remarks. Throughout this work we use Planck units in which $c=G=\hbar=k_{B}=1$.

\section{SPHERICALLY SYMMETRIC BLACK HOLE IN EMGB GRAVITY}

In the case of the EGB gravity minimally coupled to the electromagnetic field, the matter component of the action (5) is given by

$$
L_{\text {matter }}=F_{\alpha \beta} F^{\alpha \beta}, \quad F_{\alpha \beta}=A_{\beta, \alpha}-A_{\alpha, \beta} .
$$

Spherically symmetric black holes of the EMGB theory have been investigated very intensively as possible scenarios for the realization of the low energy limit of certain string theories. A particular solution which contains as a special case a black hole spacetime was

obtained in [35] (see also [36 38]) by using the following 5D static spherically symmetric line element

$$
d s^{2}=-f(r) d t^{2}+\frac{d r^{2}}{f(r)}+r^{2} d \Omega_{3}^{2}
$$


where $d \Omega_{3}^{2}$ is the metric of a $3 \mathrm{D}$ hypersurface with constant curvature $6 k$ which has the explicit form

$$
d \Omega_{3}^{2}= \begin{cases}d \theta_{1}^{2}+\sin ^{2} \theta_{1}\left(d \theta_{2}^{2}+\sin ^{2} \theta_{2} d \theta_{3}^{2}\right), & (k=1) \\ d \theta_{1}^{2}+\sinh ^{2} \theta_{1}\left(d \theta_{2}^{2}+\sin ^{2} \theta_{2} d \theta_{3}^{2}\right), & (k=-1) \\ \alpha^{-1} d x^{2}+d \phi_{1}^{2}+d \phi_{2}^{2}, & (k=0) .\end{cases}
$$

Here, the coordinate $x$ has the dimension of length while the angular coordinates $\left(\theta_{1}, \theta_{2}\right) \in$ $[0, \pi]$ and $\left(\theta_{3}, \phi_{1}, \phi_{2}\right) \in[0,2 \pi]$.

A particular solution is given by the metric function

$$
f(r)=k+\frac{r^{2}}{4 \alpha}\left[1 \pm \sqrt{1+\frac{8 \alpha(M+2 \alpha|k|)}{r^{4}}-\frac{8 \alpha Q^{2}}{3 r^{6}}}\right],
$$

where the geometric mass $M+2 \alpha|k|$ is different from that of Einstein gravity for $k= \pm 1$. This solution is well defined if the expression within the square root is positive definite. For the solution (9) of the EMGB theory to describe a black hole it is necessary that the condition $f(r)=0$ be satisfied. For the special case $k=+1$, the roots of this equation are

$$
r_{ \pm}=\frac{1}{2}\left[\sqrt{M+\frac{2 Q}{\sqrt{3}}} \pm \sqrt{M-\frac{2 Q}{\sqrt{3}}}\right]
$$

independently of the value of the coupling constant $\alpha$. It turns out that in some cases these radii determine naked singularities [39]. However, the specific case with $\alpha<0$ and $k=+1$ corresponds to a solution which is asymptotically de Sitter, and represents a black hole with an event horizon situated at

$$
r_{H}=\frac{1}{2}\left[\sqrt{M+\frac{2 Q}{\sqrt{3}}}+\sqrt{M-\frac{2 Q}{\sqrt{3}}}\right],
$$

provided $\left(\frac{Q}{M}\right)^{2} \leq \frac{3}{4}$. In this section, we will limit ourselves to the study of this black hole spacetime.

It is interesting to mention that this specific black hole solution is asymptotically de Sitter although the cosmological constant does not appear explicitly in the action (5). This is a particular characteristic of the EGB theory in five dimensions [39]. Moreover, the fact that the radius of the event horizon does not depend on the value of the coupling constant $\alpha$ leads to interesting thermodynamic consequences. In fact, we will see that if we use the Bekenstein-Hawking entropy relationship the thermodynamics of the black hole differs completely from the one obtained by using a modified entropy in which the coupling constant appears explicitly. 


\section{A. Analysis with the Bekenstein-Hawking entropy relation}

Since the surface area of the event horizon is given by

$$
A=r_{H}^{3} \int_{\theta=0}^{\pi} \int_{\phi=0}^{\pi} \int_{\psi=0}^{2 \pi} \sin ^{2} \theta \sin \phi d \theta d \phi d \psi=2 \pi^{2} r_{H}^{3}
$$

the Bekenstein-Hawking entropy of the black hole is $\frac{k_{B} A}{4 G \hbar}=\frac{k_{B} \pi^{2}}{2 G \hbar} r_{H}^{3}$ [40]. Choosing the constants appropriately, the entropy takes the form $S=r_{H}^{3}$, representing the fundamental equation that contains all the thermodynamic information. In the mass representation, $M=M(S, Q)$, for the black hole solution presented above this fundamental equation can be rewritten as

$$
M=S^{\frac{2}{3}}+\frac{1}{3} \frac{Q^{2}}{S^{\frac{2}{3}}} .
$$

\section{Thermodynamics}

Using the energy conservation law of the black hole (i. e., $d M=T d S+\phi d Q$ ), one obtains the temperature and electric potential of the black hole on the event horizon as

$$
T=\frac{2}{9} \frac{3 S^{\frac{4}{3}}-Q^{2}}{S^{\frac{5}{3}}},
$$

and

$$
\phi=\frac{2}{3} \frac{Q}{S^{\frac{2}{3}}}
$$

In the positive domain $\left(S^{4 / 3}>Q^{2} / 3\right)$, the temperature increases rapidly as a function of the entropy $S$ until it reaches its maximum value at $S^{4 / 3}=5 Q^{2} / 3$. Then, as the entropy increases, the temperature becomes a monotonically decreasing function. This behavior is shown in Fig. 1,

According to Davies [41], the phase transition structure of the black hole can be derived from the behavior of the heat capacity. Strictly speaking, this implies that first we must introduce the concept of "heat", say $Q_{\text {heat }}$, for a black hole. This is a complicated question that has not been answered so far, in particular, due to the lack of a physically reasonable statistical model for black holes [51]. For this reason, we use here the analogy with ordinary thermodynamics as follows. Using the the thermodynamic potential $M(S, Q)$ in which the

first law of thermodynamics is expressed as $d M=T d S+\phi d Q$, we define the "heat" through the relationship $d Q_{\text {heat }} \equiv T d S$ so that $d M=d Q_{\text {heat }}+\phi d Q$. Then, following the standard 

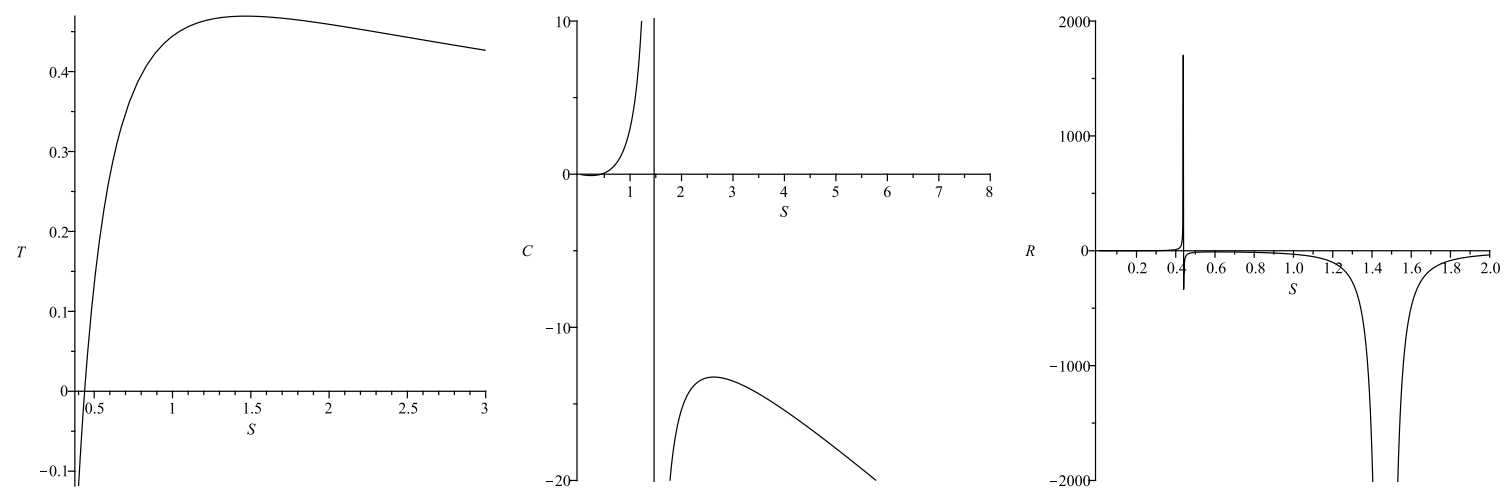

FIG. 1: Temperature $T$, heat capacity $C_{Q}$ and thermodynamic curvature $R$ of a charged black hole in EGB theory as a function of the Bekenstein-Hawking entropy $S$ for $Q=1$. The curvature singularities are located at the points $T=0$ and $C_{Q} \rightarrow \infty$.

approach of ordinary thermodynamics, we introduce the heat capacity $C_{Q} \equiv\left(\frac{\partial Q_{\text {heat }}}{\partial T}\right)_{Q}=$ $\left(\frac{\partial M}{\partial T}\right)_{Q}$ that in this case is given by

$$
C_{Q}=-3 S\left(\frac{3 S^{\frac{4}{3}}-Q^{2}}{3 S^{\frac{4}{3}}-5 Q^{2}}\right)
$$

In the physical region with $3 S^{4 / 3}-Q^{2}>0$, i. e., the region with positive temperature, the heat capacity is positive in the interval $Q^{2}<3 S^{4 / 3}<5 Q^{2}$, indicating that the black hole is stable in this region. At the maximum value of the temperature which occurs at $3 S^{4 / 3}-$ $5 Q^{2}=0$, the heat capacity diverges and changes spontaneously its sign from positive to negative. This indicates the presence of a second order phase transition which is accompanied by a transition into a region of instability (see Fig[1).

Usually, when applied to ordinary thermodynamic systems, the above analysis is associated with a particular statistical ensemble, once the internal energy of the system is well defined. In the case of black holes, however, there are several possibilities to define the internal energy. For this reason, sometimes the potential $M(S, Q)$, or equivalently $S(M, Q)$, is associated with the microcanonical ensemble [42], the canonical ensemble with fixed potential [43] and, in principle, one could also associate it with the grand canonical ensemble because the system is allowed to exchange charge. Here, we will use this last option.

Using this notation, we can say that the phase transition structure we have found above for the EMGB black hole is based upon the analysis of the grand canonical ensemble. On the other hand, it is known that the critical points of the heat capacities may depend 
on the ensemble. In the case of the EMGB black hole we are considering here, from the thermodynamic potential $M(S, Q)$ we can define the potentials

$$
H \equiv M-\phi Q, \quad F \equiv M-T S, \quad G \equiv M-T S-\phi Q
$$

that are usually denoted as the enthalpy, the Helmholtz free energy, and the Gibbs free energy, respectively. The enthalpy satisfies the first law of thermodynamics, $d H=T d S-$ $Q d \phi$, and can be considered as determining the canonical ensemble. Then, if we assume again that the "heat" of the black hole is defined by $d Q_{\text {heat }}=T d S$, the heat capacity for fixed $\phi$ is given by

$$
C_{\phi} \equiv\left(\frac{\partial Q_{\text {heat }}}{\partial T}\right)_{\phi}=\left(\frac{\partial H}{\partial T}\right)_{\phi}=-3 S .
$$

We see that in this ensemble the heat capacity is always negative for $S>0$, indicating that the black hole is unstable. In the limit $S \rightarrow 0$, the thermodynamic description breaks down, because it also implies that $H \rightarrow 0$ and $M \rightarrow \infty$ [52].

Consequently, the phase structure predicted by $C_{\phi}$ is completely different from that of $C_{Q}$. In this context, it is interesting to investigate the behavior of the thermodynamic potentials at the points of phase transitions. From Eq.(13), we can calculate explicitly all the potentials and investigate their properties. In Fig. 2, we illustrate the behavior of the potentials near the point where the heat capacity $C_{Q}$ diverges. At this point, all the potentials are well behaved and only the Helmholtz energy possesses a minimum. This behavior is illustrated in the right plot of Fig. 22 that shows the stable minima of the Helmholtz energy for different values of the charge. We conclude that the phase transitions corresponding to divergences of $C_{Q}$ occur in a region of stability of $F$. Nevertheless, after the phase transition the black hole becomes unstable.

These are the main features of the thermodynamic behavior of the charged spherically symmetric black hole (9).

\section{Geometrothermodynamics}

For our geometrothermodynamic approach to black hole thermodynamics all what is needed is the fundamental equation, $M=M(S, Q)$ as given in Eq.(13). Then, from the general metric (3) with $\Phi=M$ and $E^{a}=(S, Q)$ we obtain the thermodynamic metric of 

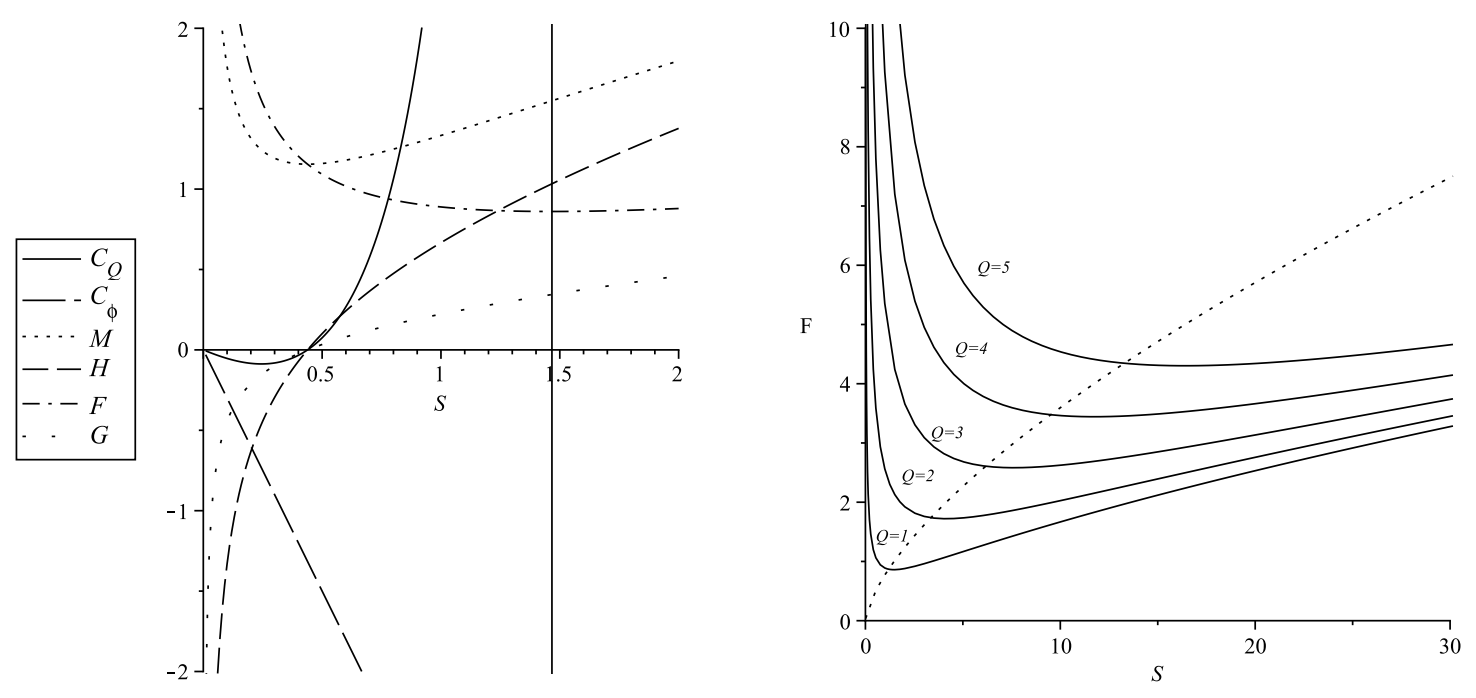

FIG. 2: Left plot: Heat capacities and thermodynamic potentials of the EMGB black hole for a fixed value of the charge $Q=1$. Right plot: The Helmholtz free energy for different values of the charge $Q$. The dotted curve denotes the location of the minima where the phase transitions occur.

the equilibrium manifold:

$$
g=\frac{4}{27} \frac{3 S^{4 / 3}-Q^{2}}{S^{4 / 3}}\left(\frac{3 S^{4 / 3}-5 Q^{2}}{9 S^{2}} d S^{2}+d Q^{2}\right)
$$

The corresponding scalar curvature is given by

$$
R=-\frac{243 S^{8 / 3}}{\left(3 S^{4 / 3}-Q^{2}\right)\left(3 S^{4 / 3}-5 Q^{2}\right)^{2}} .
$$

A first singularity is situated at the roots of the equation $3 S^{4 / 3}-Q^{2}=0$, i. e., at the points where the temperature vanishes. The second singularity corresponds to the roots of $3 S^{4 / 3}-5 Q^{2}=0$. According to the expression for the heat capacity $C_{Q}$ given in Eq.(16), these are exactly the points where phase transitions take place and the temperature reaches its maximum value. It follows that the geometrothermodynamic curvature of the metric (19) reproduces correctly the thermodynamic behavior near the points of zero temperature as well as near the points of phase transitions (see Fig,1). The above results show that GTD correctly describes the thermodynamic properties of the black hole under consideration in the grand canonical ensemble in which $C_{Q}$ was considered.

Notice that the curvature of the metric (19) cannot reproduce the phase transition structure predicted by $C_{\phi}$, because it represents a different definition of phase transitions based on the use of a different ensemble, namely, the canonical ensemble. Nevertheless, in GTD 
we can also reproduce the results obtained from the analysis of $C_{\phi}$ by using the fact that the general thermodynamic metric (3i) can be applied to any potential in any representation. Below we will show this explicitly.

Consider the canonical ensemble with the thermodynamic potential

$$
H \equiv M-\phi Q=S^{2 / 3}\left(1-\frac{3}{4} \phi^{2}\right),
$$

in which the first law reads $d H=T d S-Q d \phi$. Then, the corresponding temperature can be expressed as

$$
T=\frac{2}{3} S^{-1 / 3}\left(1-\frac{3}{4} \phi^{2}\right),
$$

and the heat capacity $C_{\phi}$, as mentioned above, is always negative for $S>0$, i. e., the black hole is unstable. Since the fundamental equation in this ensemble is $H=H(S, \phi)$, we choose the thermodynamic potential $\Phi=H$ and the independent thermodynamic variables $E^{a}=(S, \phi)$ to construct the thermodynamic metric of the equilibrium manifold. Then, introducing Eq.(21) into the metric (3), we get

$$
g=\left(1-\frac{3}{4} \phi^{2}\right)\left[\frac{4}{27} S^{-2 / 3}\left(1-\frac{3}{4} \phi^{2}\right) d S^{2}-S^{4 / 3} d \phi^{2}\right],
$$

from which we obtain the curvature scalar

$$
R=\frac{3}{S^{4 / 3}\left(1-\frac{3}{4} \phi^{2}\right)^{3}} .
$$

In GTD, we interpret the non-vanishing of the curvature as due to the presence of thermodynamic interaction in the system, regardless of its stability properties. In this case, we see that a non-zero curvature describes the thermodynamics of a completely unstable black hole. Indeed, from the above expression for the curvature scalar, it follows that there is a first singularity for $S \rightarrow 0$ that corresponds to the limit $C_{\phi} \rightarrow 0$ at which, as mentioned above, the thermodynamic description breaks down. The second singularity is located at $\phi^{2}=4 / 3$ and corresponds to the limit of vanishing temperature. We conclude that the curvature singularities of the equilibrium manifold correspond to the locations where the thermodynamic description of the black hole in this ensemble breaks down.

The above results corroborate the well-known fact that the phase transition structure depends on the chosen ensemble. In this context, it is interesting to investigate the thermodynamic properties that follow from the study of the Gibbs function. From Eqs.(14) and 
(15), we obtain

$$
S=\frac{8}{27 T^{3}}\left(1-\frac{3}{4} \phi^{2}\right)^{3}, \quad Q=\frac{2 \phi}{3 T^{2}}\left(1-\frac{3}{4} \phi^{2}\right)^{2}
$$

so that

$$
G=M-T S-\phi Q=\frac{4}{27 T^{2}}\left(1-\frac{3}{4} \phi^{2}\right)^{2} .
$$

Thus, the Gibbs energy is a well-behaved function in the interval $\phi^{2}<4 / 3$ of positive temperature. To study this ensemble in GTD, we consider the thermodynamic metric (3) with $\Phi=G$ and $E^{a}=(T, \phi)$, and introduce in the resulting metric the fundamental equation (26). Then, we obtain the expression

$$
g=\frac{4}{81 T^{4}}\left(1-\frac{3}{4} \phi^{2}\right)^{4}\left[\frac{16}{3 T^{2}}\left(1-\frac{3}{4} \phi^{2}\right)^{2} d T^{2}+\left(4-15 \phi^{2}\right) d \phi^{2}\right],
$$

for which the following curvature scalar can be derived

$$
R=-\frac{729 T^{4}}{\left(1-\frac{3}{4} \phi^{2}\right)^{5}\left(4-15 \phi^{2}\right)^{2}} .
$$

The first curvature singularity appears at $\phi^{2}=4 / 3$, and corresponds to the limit $T \rightarrow 0$ at which the thermodynamic approach breaks down. The second singularity at $\phi^{2}=4 / 15$, which coincides with the point where the second derivative of $G$ vanishes, indicates the presence of a second-order phase transition. This result is in accordance with the one obtained for the thermodynamic potential $M=M(S, Q)$ in Eq.(20). Indeed, using the Eqs.(25), one can show that $3 S^{4 / 3}-5 Q^{2} \propto 4-15 \phi^{2}$, and this is exactly the term in the denominator of (20) that vanishes as $C_{Q} \rightarrow \infty$.

The thermodynamic geometry of this black hole was also studied in [35] using the Ruppeiner geometry. It turns out that the Ruppeiner metric is flat in this case and, consequently, cannot reproduce the behavior at the places where phase transitions occur or the temperature vanishes.

\section{B. Geometrothermodynamics with a modified entropy relation}

Usually the entropy of black holes satisfies the so-called area formula, i.e, the black hole entropy equals one-quarter of the horizon area. In gravity theories in higher dimensions and with higher powered curvature terms, however, the entropy of black holes does not necessarily satisfy the area formula and other possibilities can be considered to define entropy. For 
instance, in [44] a simple method was suggested to obtain the black hole entropy, by assuming that black holes, considered as genuine thermodynamic systems, must obey the first law of thermodynamics. That is, we suppose that a black hole solution, parameterized by the mass $M$ or, alternatively, by the outer horizon radius $r_{H}$, and the temperature $T$, satisfies the first law of thermodynamics $d M=T d S+\mu_{i} d Q^{i}$, where $Q^{i}$ are the additional charges of the black hole and $\mu_{i}$ are the corresponding chemical potentials. If the mass and the temperature can be calculated by using standard methods, the integration of the first law yields the modified entropy formula

$$
S=\int_{0}^{r_{H}} T^{-1}\left(\frac{\partial M}{\partial r_{H}}\right)_{Q_{i}} d r_{H}+S_{0}
$$

where the additive integration constant $S_{0}$ can be fixed by imposing the condition that the entropy goes to zero in the case of an extreme black hole or when the area of the horizon vanishes. Notice that in the integration (29) the charges $Q_{i}$ must be considered as constants. In [45, 46], the modified entropy was computed for an $n$-dimensional generalization of the black hole solution (9) with the result

$$
S=\frac{\Omega_{K} r_{H}^{n-2}}{4 G}\left[1+\frac{2 \tilde{\alpha} k(n-2)}{(n-4) r_{H}^{2}}\right]
$$

where $\Omega_{k}$ denotes the spatial volume element, and $\tilde{\alpha}=(n-2)(n-3) \alpha$. In the case $n=5$, we are considering here, the modified entropy reduces to

$$
S=r_{H}^{3}+6 k \tilde{\alpha} r_{H}
$$

where suitable units were chosen and we set $S_{0}=0$ for simplicity. Notice that the contribution of the correction term vanishes for $k=0$ so that the GB term has no effect on the expression for the entropy which reduces in this case to the standard area formula. Moreover, the modified entropy formula does not contain the additional charges $Q_{i}$ explicitly, but only implicitly through the horizon radius $r_{H}$. So we can assume the validity of the modified entropy (31) regardless of the nature of the additional charges.

For the black hole solution (9) the modified entropy formula (with $k=1$ ) gives

$$
S=\frac{1}{8}\left[\sqrt{M+\frac{2 Q}{\sqrt{3}}}+\sqrt{M-\frac{2 Q}{\sqrt{3}}}\right]^{3}+3 \tilde{\alpha}\left[\sqrt{M+\frac{2 Q}{\sqrt{3}}}+\sqrt{M-\frac{2 Q}{\sqrt{3}}}\right] .
$$

In this case the fundamental equation is of the form $S=S(M, Q)$ and cannot be rewritten explicitly as $M=M(S, Q)$. This means that for the further analysis we must use the entropy 

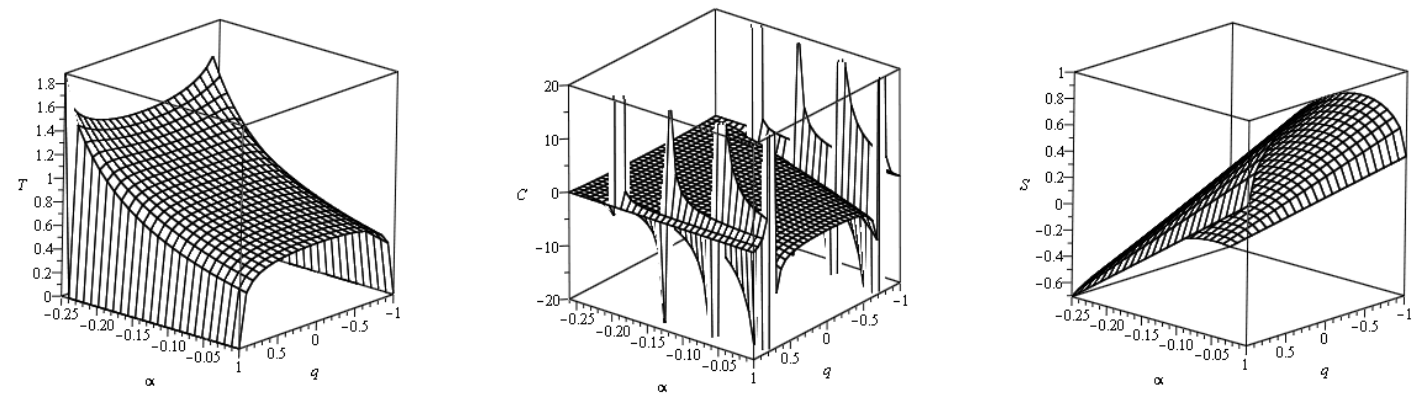

FIG. 3: Modified temperature, heat capacity $C_{Q}$, and entropy as functions of the specific charge $q$ and the coupling parameter $\tilde{\alpha}$ for a fixed value of the mass $M=1$.

representation. This is not a problem for the formalism of GTD which can be applied to any arbitrary representation. In fact, for the entropy representation we only need to consider the fundamental one-form as

$$
\Theta_{S}=d S-\frac{1}{T} d M+\frac{\phi}{T} d Q
$$

so that the thermodynamic potential is $\Phi=S$, the coordinates of the equilibrium manifold are $E^{a}=(M, Q)$, and the equilibrium conditions become

$$
\frac{1}{T}=\frac{\partial S}{\partial M}, \quad \frac{\phi}{T}=-\frac{\partial S}{\partial Q} .
$$

From the above expressions one obtains the temperature and electric potential of the black hole on the event horizon as

$$
\begin{gathered}
T=\frac{8}{3} \frac{\sqrt{M\left(1-q^{2}\right)}}{\left(4 \tilde{\alpha}+M+M \sqrt{1-q^{2}}\right)(\sqrt{1+q}+\sqrt{1-q})}, \\
\phi=\frac{2}{\sqrt{3}} \frac{\sqrt{1+q}-\sqrt{1-q}}{\sqrt{1+q}+\sqrt{1-q}},
\end{gathered}
$$

where $q=\frac{2 Q}{\sqrt{3} M}$ represents a rescaled specific charge that satisfies the condition $q^{2} \leq 1$. Furthermore, to find out the phase transitions structure we must find the points where the heat capacity $\left(C_{Q}=T \partial S /\left.\partial T\right|_{Q}=-(\partial S / \partial M)^{2} /\left.\left(\partial^{2} S / \partial M^{2}\right)\right|_{Q}\right)$

$$
C_{Q}=-\frac{3}{4} \frac{\sqrt{M} \sqrt{1-q^{2}}(\sqrt{1+q}+\sqrt{1-q})\left(4 \tilde{\alpha}+M+M \sqrt{1-q^{2}}\right)^{2}}{M\left(1-3 q^{2}\right)+(4 \tilde{\alpha}+M) \sqrt{1-q^{2}}-8 \tilde{\alpha}}
$$

diverges. Since the coupling constant $\tilde{\alpha}$ is negative, the temperature function turns out to be positive definite only for certain ranges of $\tilde{\alpha}$ and $q$. In Fig 3 , we choose a particular range 

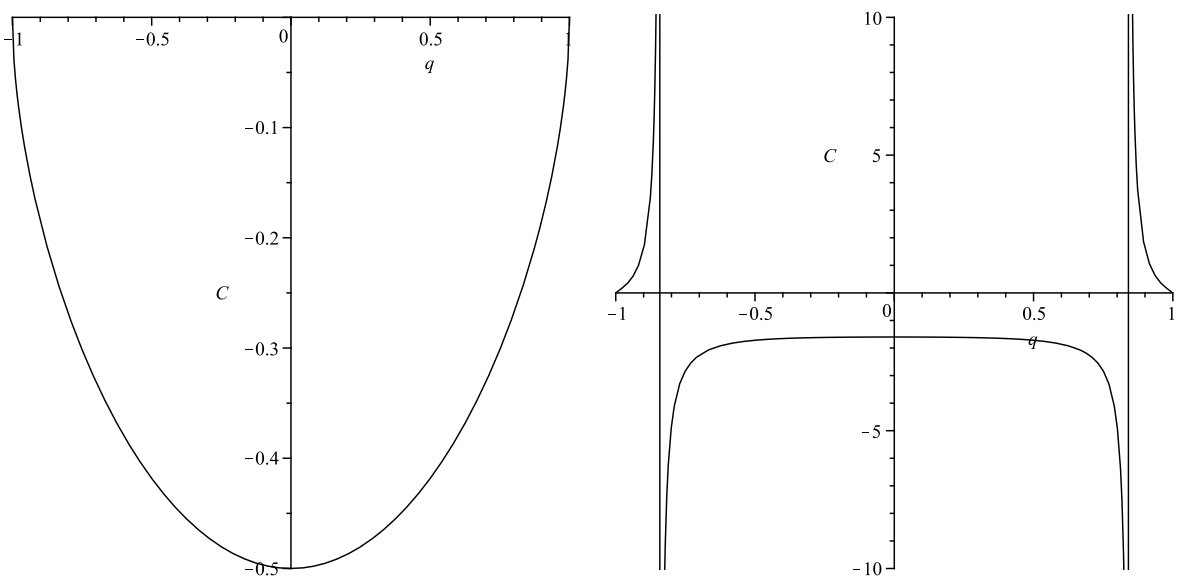

FIG. 4: The heat capacity $C_{Q}$ as a function of the specific charge $q$ for $\tilde{\alpha}=-1 / 4$ (left plot) and $\tilde{\alpha}=-1 / 10$ (right plot). In both cases the mass is $M=1$.

of values of $\tilde{\alpha} \in[-1 / 4,0]$ in which the modified temperature is positive. We also plot the modified heat capacity and entropy in the same range of values. Notice that the entropy is not positive definite in this interval; however, it is possible to choose the arbitrary constant $S_{0}$ in Eq.(29) so that the modified entropy is always positive and the expressions for the modified temperature and heat capacity remain unchanged.

An interesting result of using the modified entropy is that the phase transition structure now depends on the value of the coupling constant $\tilde{\alpha}$. For instance, if we choose it as $\tilde{\alpha}=$ $-1 / 4$, the heat capacity is as illustrated in Fig. 团(left plot). In this case, the heat capacity is represented by a negative smooth function with no singularities in the interval $-1 \leq q \leq 1$, indicating that the black hole is a completely unstable thermodynamic system with no phase transition structure. This behavior changes drastically, if we choose a different value of the coupling constant. In fact, Fig 4 (right plot) illustrates the singular behavior of the heat capacity in the case $\tilde{\alpha}=-1 / 10$. We can see that at $q \approx \pm 0.82$, the black hole undergoes a second order phase transition. In the interval $-0.82 \leq q \leq 0.82$, the configuration is unstable because the heat capacity is negative. Outside this interval, however, the black hole is stable. We conclude that the coupling constant $\tilde{\alpha}$ can induce a second order phase transition in an unstable black hole in such a way that the resulting configuration is a stable black hole for certain values of the specific charge $q$.

Now we investigate this black hole configuration in the context of GTD. As mentioned above, the coordinates of the equilibrium manifold are $E^{a}=(M, Q)$ and the thermody- 

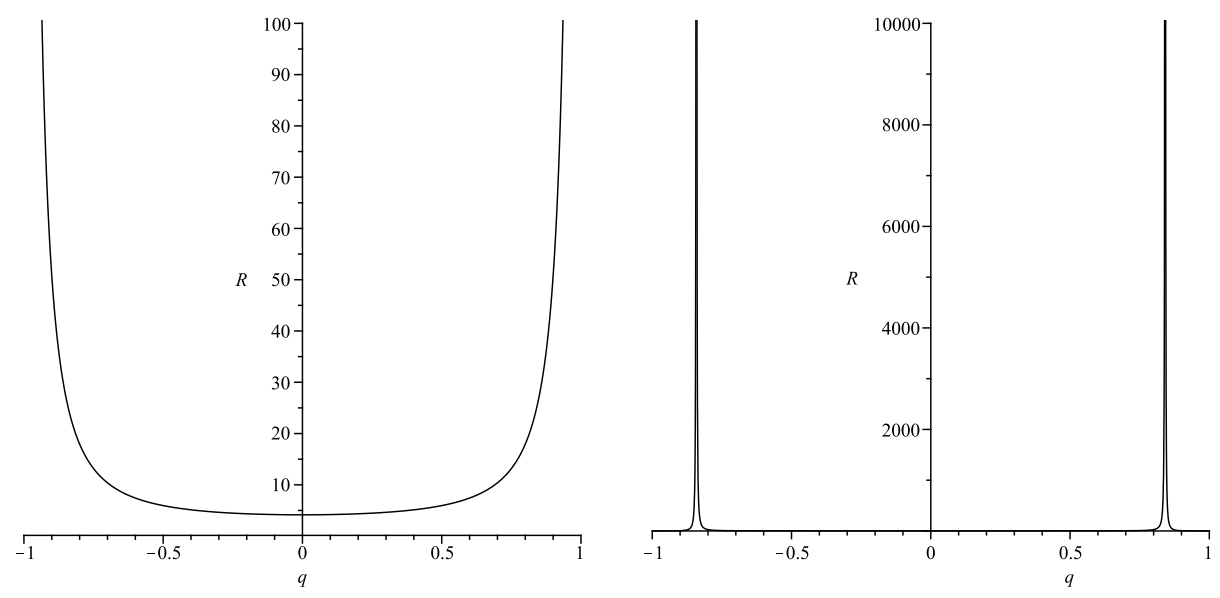

FIG. 5: Thermodynamic curvature as a function of the rescaled specific charge $q$. For $\tilde{\alpha}=-1 / 4$ (left plot) the curvature is completely regular, except at $q= \pm 1$. Curvature singularities appear for $q \approx 0.82$ and $\tilde{\alpha}=-1 / 10$ (right plot).

namic potential $\Phi=S$ is given by means of the fundamental equation (32). Then, the thermodynamic metric for the equilibrium manifold $\mathcal{E}$ is given by

$$
\begin{aligned}
g=-\frac{3 r_{H}\left(r_{H}^{2}+2 \tilde{\alpha}\right)}{8 \sqrt{1-q^{2}} M^{2}}\{ & \frac{3 r_{H}\left[2 r_{H}^{2}-3 q^{2} M+4 \tilde{\alpha}\left(\sqrt{1-q^{2}}-2\right)\right]}{2\left(1-q^{2}\right)^{3 / 2}} d M^{2} \\
& \left.+\sqrt{M}\left[\frac{4 \tilde{\alpha}+(2+q) M}{(1+q)^{3 / 2}}+\frac{4 \tilde{\alpha}+(2-q) M}{(1-q)^{3 / 2}}\right] d Q^{2}\right\} .
\end{aligned}
$$

The behavior of the corresponding scalar curvature is shown in Fig 5 for two different values of the coupling constant. The plot on the left shows the case $\tilde{\alpha}=-1 / 4$ and corresponds to the case of an unstable configuration as shown in Fig. 4 (left plot). We can see that the curvature is represented by a smooth function that is free of singularities in the entire domain of the specific charge, except at $q= \pm 1$ where the temperature vanishes (see Fig 3 ). The plot on the right illustrates the behavior for $\tilde{\alpha}=-1 / 10$ and shows two curvature singularities at $q \approx \pm 0.82$ which are the points where the phase transition occurs (see right plot in Fig 4). In this case, it is also possible to show that an additional curvature singularity (not plotted) exists in the limiting case $q \rightarrow \pm 1$, indicating the blow up of the approach as $T \rightarrow 0$. 


\section{SPHERICALLY SYMMETRIC BLACK HOLE IN EMGB GRAVITY WITH COSMOLOGICAL CONSTANT}

In the case of the Einstein-Maxwell-Gauss-Bonnet theory with cosmological constant, the matter component of the action (5) is given by

$$
L_{\text {matter }}=F_{\alpha \beta} F^{\alpha \beta}-2 \Lambda, \quad F_{\alpha \beta}=A_{\beta, \alpha}-A_{\alpha, \beta},
$$

where $\Lambda$ is the cosmological constant, and $F_{\alpha \beta}$ represents the electromagnetic Faraday tensor.

A five dimensional spherically symmetric solution in EMGB gravity with $\Lambda$ was obtained by Wiltshire [47], using the metric ansatz (17) with $k=+1$ and the metric function

$$
f(r)=1+\frac{r^{2}}{4 \alpha}-\frac{r^{2}}{4 \alpha} \sqrt{1+\frac{8 \alpha M}{r^{4}}-\frac{8 \alpha Q^{2}}{3 r^{6}}+\frac{4 \alpha \Lambda}{3}} .
$$

The two parameters $M(>0)$ and $Q$ are identified as the mass and electric charge of the

system. The limit of vanishing cosmological constant generates a solution contained in Eq.(9) with the minus sign in front of the square root and a redefined mass parameter. In this limit, however, the resulting solution does not describe a black hole, but a naked singularity.

In order for the solution (40) to describe a black hole spacetime, it is necessary that the expression inside the square root be positive and the function $f(r)$ vanish on the horizon radius, i. e.,

$$
1+\frac{8 \alpha M}{r_{H}^{4}}-\frac{8 \alpha Q^{2}}{3 r_{H}^{6}}+\frac{4 \alpha \Lambda}{3}>0, \quad 1+\frac{r_{H}^{2}}{4 \alpha}-\frac{r_{H}^{2}}{4 \alpha} \sqrt{1+\frac{8 \alpha M}{r_{H}^{4}}-\frac{8 \alpha Q^{2}}{3 r_{H}^{6}}+\frac{4 \alpha \Lambda}{3}}=0 .
$$

Moreover, to guarantee that the mass of the black hole is always positive (see below) we

must demand that the coupling constant $\alpha$ be positive and the cosmological constant $\Lambda$ be positive definite. In this section we will limit ourselves to this range of parameters, so that the black hole determined by the function (40) turns out to be asymptotically anti de Sitter.

\section{A. Analysis with the Bekenstein-Hawking entropy relation}

The condition $f\left(r_{H}\right)=0$ implies that

$$
\frac{\Lambda}{3} r_{H}^{6}-2 r_{H}^{4}+2(M-2 \alpha) r_{H}^{2}-\frac{2}{3} Q^{2}=0 .
$$


Moreover, as we mentioned in Sec.(IIA), with the appropriate choice of units the BekensteinHawking entropy of the black hole is given by $S=r_{H}^{3}$. Then, the corresponding thermodynamic fundamental equation in the mass representation becomes

$$
M=2 \alpha+S^{2 / 3}+\frac{Q^{2}}{3 S^{2 / 3}}-\frac{\Lambda}{6} S^{4 / 3} .
$$

Notice that to guarantee the positiveness of the mass, we must assume that $\alpha>0$ and $\Lambda<0$.

\section{Thermodynamics}

Using the energy conservation law of the black hole (i. e. $d M=T d S+\phi d Q$ ), one obtains the temperature and electric potential of the black hole on the event horizon as

$$
T=\frac{2}{9} \frac{3 S^{4 / 3}-\Lambda S^{2}-Q^{2}}{S^{5 / 3}},
$$

and

$$
\phi=\frac{2 Q}{3 S^{\frac{2}{3}}} .
$$

Now, for the grand canonical ensemble the heat capacity has the form

$$
C_{Q}=3 S\left(\frac{3 S^{\frac{4}{3}}-\Lambda S^{2}-Q^{2}}{5 Q^{2}-3 S^{\frac{4}{3}}-\Lambda S^{2}}\right) .
$$

The expression for the temperature (44) shows that it is positive only in the range $3 S^{4 / 3}-\Lambda S^{2}>Q^{2}$. Consequently, the heat capacity can take either positive or negative values, indicating the possibility of stable and unstable states for the black hole. In fact, the expression for the heat capacity exhibits a very rich structure in the range where the temperature is positive. In Fig [6 a particular range was chosen to show the behavior of the temperature and the heat capacity. The plot on the right shows for the particular value $Q=1 / 2$ two different phase transitions at $S \approx 0.6$ and $S \approx 4.9$. The first one corresponds to a transition from a stable state $\left(C_{Q}>0\right)$ to an unstable state $\left(C_{Q}<0\right)$. The second one represents a second order phase transition in which the black hole becomes a stable system again.

Let us now consider the thermodynamic potential

$$
H=M-\phi Q=2 \alpha+S^{2 / 3}-\frac{3}{4} \phi^{2} S^{2 / 3}-\frac{\Lambda}{6} S^{4 / 3} .
$$



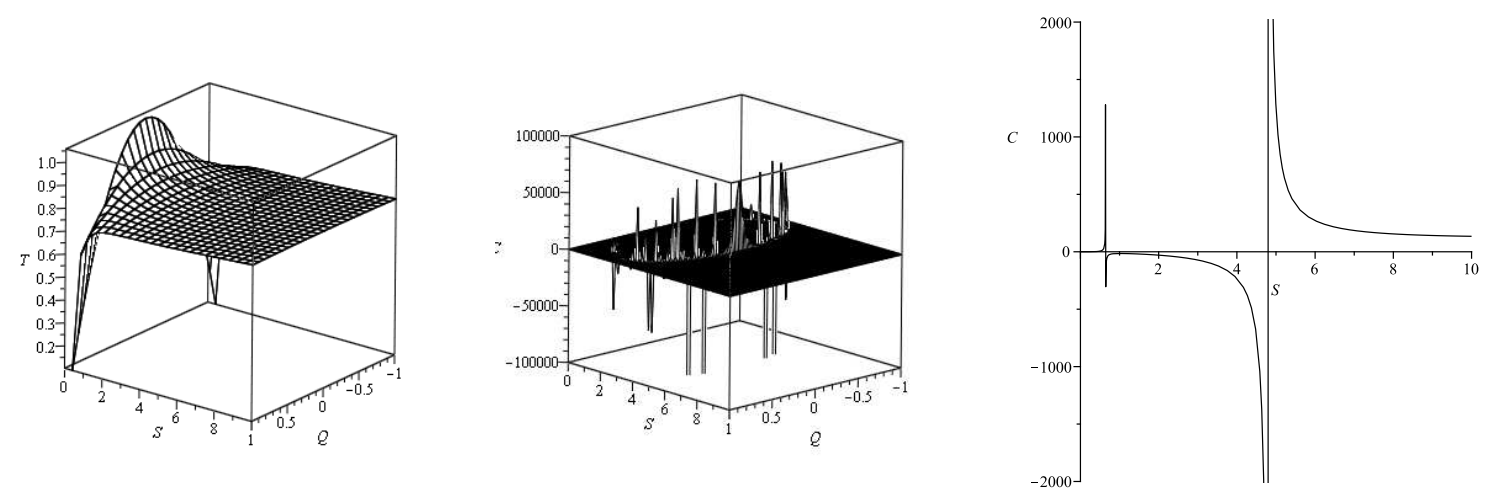

FIG. 6: Behavior of the temperature and the heat capacity $C_{Q}$ in the range $Q \in[-1,1]$ and $S \in[0,10]$ for a fixed value of the cosmological constant $\Lambda=-1$. The right plot shows the details of the phase transition structure for the particular charge $Q=1 / 2$.

for the canonical ensemble in which the first law of thermodynamics reads $d H=d Q_{\text {heat }}-Q d \phi$ with $d Q_{\text {heat }}=T d S$, as before. In this ensemble, we can define the heat capacity at fixed electric potential, i. e.

$$
C_{\phi} \equiv\left(\frac{\partial H}{\partial T}\right)_{\phi}=-3 S \frac{12 S^{1 / 3}-9 \phi^{2} S^{1 / 3}-4 \Lambda S}{12 S^{1 / 3}-9 \phi^{2} S^{1 / 3}+4 \Lambda S}
$$

whose behavior strongly depends on the value of the cosmological constant. Figure 7 shows that for a given value of $\Lambda$, the heat capacity $C_{\phi}$ can be either positive or negative with a quite complex singularity structure. If we take into account the condition $12 S^{1 / 3}-9 \phi^{2} S^{1 / 3}-4 \Lambda S>$ 0 , which follows from the condition $T>0$, we find that stable states $\left(C_{\phi}>0\right)$ are allowed for entropies in the range

$$
S^{2 / 3}>\frac{3}{4|\Lambda|}\left(4-3 \phi^{2}\right)
$$

If we choose $\phi^{2}>4 / 3$, this condition is always satisfied for $S>0$, indicating that stable states always exist in this case. Moreover, from the heat capacity (48) we see that the roots of the equation $12 S^{1 / 3}-9 \phi^{2} S^{1 / 3}+4 \Lambda S=0$ determine the locations where second order phase transitions occur. This behavior is illustrated in Fig. 8. The left plot, where the interval has been chosen such that $T>0$ is always satisfied, shows a phase transition at $S_{c} \approx 0.61$. The black hole is stable for $S>S_{c}$, and unstable in the interval $0<S<S_{c}$. The right plot shows that for a given value of the entropy, it is possible to find a range of values for $\phi$ in which the temperature is positive definite and the heat capacity is positive, singular or negative, indicating the existence of stable and unstable black holes. 


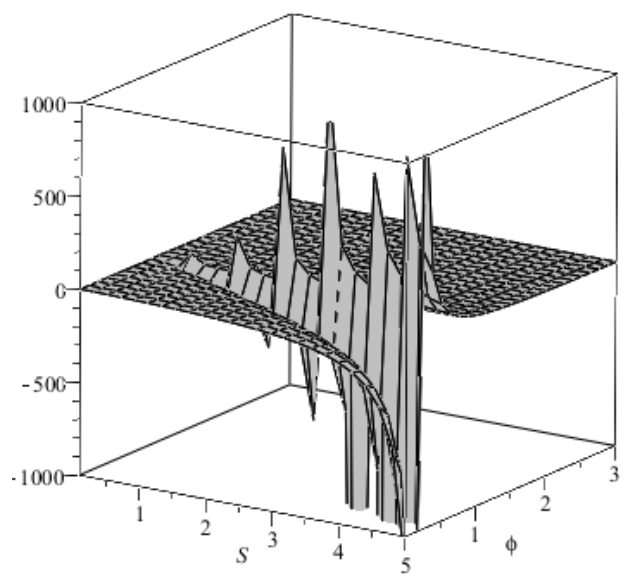

FIG. 7: The behavior of the heat capacity $C_{\phi}$ as a function of the entropy $S$ for different values of the electric potential $\phi$ and $\Lambda=-1$.
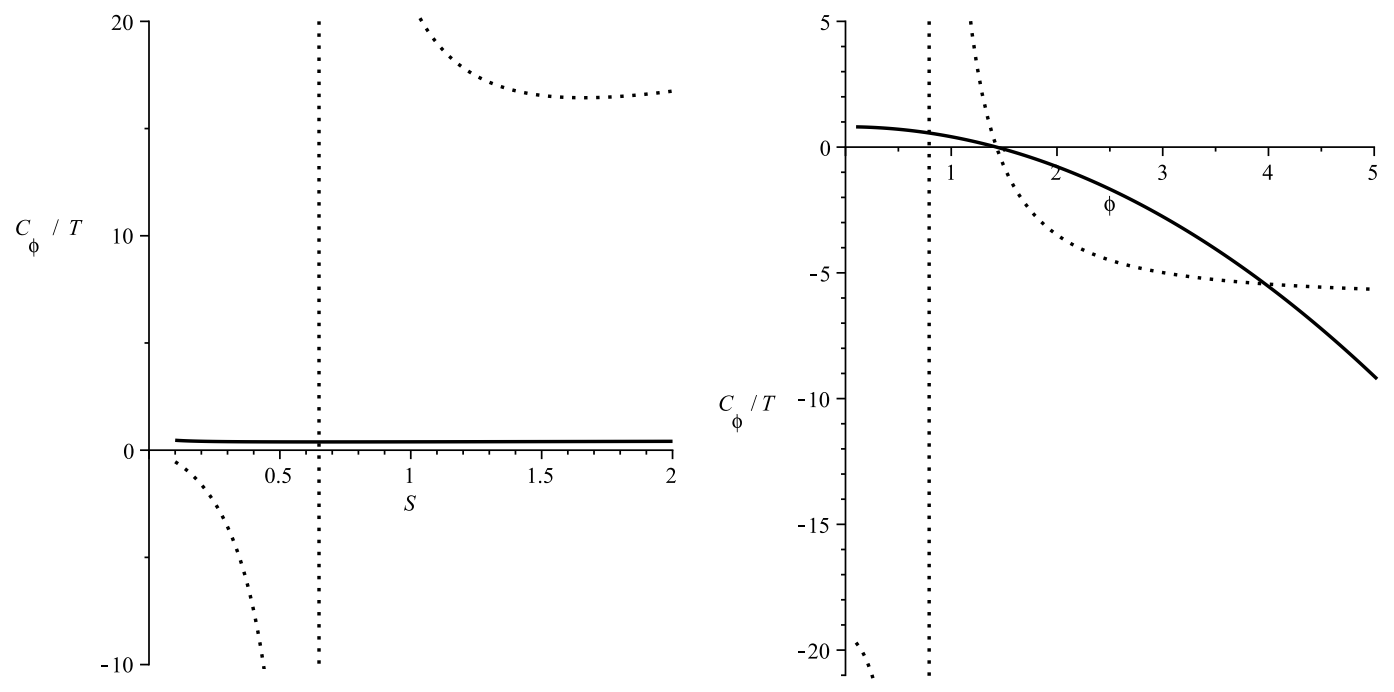

FIG. 8: The behavior of the heat capacity (dotted curve) and the temperature (solid curve) for a fixed value of the electric potential, $\phi=1$, (left plot) and for a fixed value of the entropy, $S=2$ (right plot). In both plots we set $\Lambda=-1$.

Notice that the singularities of $C_{\phi}$ are different from those of $C_{Q}$; consequently, the corresponding phase transition structures do not coincide. In fact, in Fig. 9 we show the behavior of the heat capacities and all thermodynamic potentials for this case. First, we see that at the points of phase transitions in $C_{\phi}$ all the potentials are well behaved and no critical points are observed. Moreover, the first divergence of $C_{Q}$ (at $S \approx 0.62$ ) is situated 

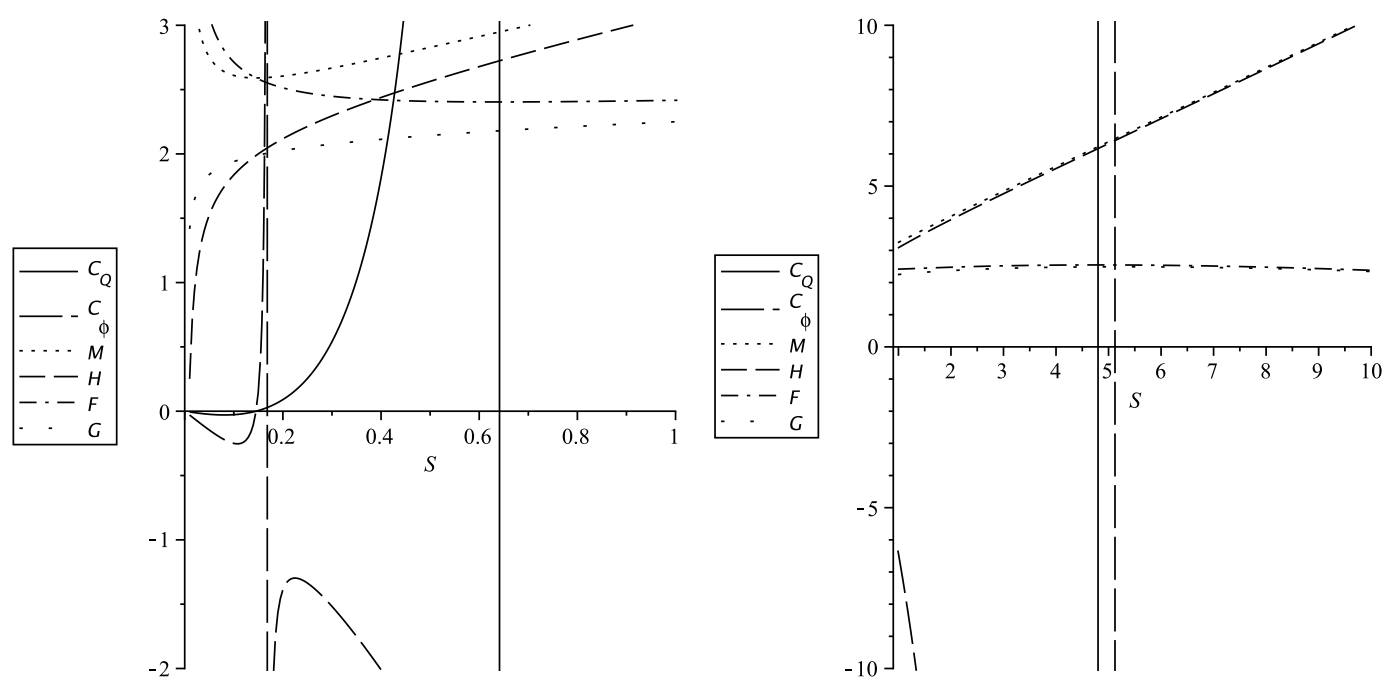

FIG. 9: Heat capacities and thermodynamic potentials in terms of the entropy for $\alpha=1, \Lambda=-1$, and $Q=1 / 2$. For clarity, two different intervals of $S$ are depicted with different scales.

on a local minimum of $F$ whereas the second singularity (at $S \approx 4.8$ ) is located on a local maximum. This situation is also illustrated in Fig. 10 where the Helmholtz free energy is plotted in terms of the entropy for different values of the electric charge. The first phase transition occurs on a metastable point of $F$ and describes a configuration in which a stable black hole transforms into an unstable one. On the contrary, the second phase transition occurs on an unstable value $F$ and corresponds to a black hole that passes from an unstable state to a stable state.

\section{Geometrothermodynamics}

In this subsection, we derive the thermodynamic metrics for the equilibrium manifold. In the case of the grand canonical ensemble, the fundamental equation is given as $M=M(S, Q)$ in Eq.(43). Then, we associate the coordinates $E^{a}=(S, Q)$ to the equilibrium manifold $\mathcal{E}$ and $\Phi=M$ is the thermodynamic potential. The thermodynamic metric (3) can then be written as

$$
g=\frac{4}{27 S^{4 / 3}}\left(3 S^{4 / 3}-\Lambda S^{2}-Q^{2}\right)\left[\frac{1}{9 S^{2}}\left(3 S^{4 / 3}+\Lambda S^{2}-5 Q^{2}\right) d S^{2}+d Q^{2}\right] .
$$

A straightforward computation results in the following scalar curvature:

$$
R=\frac{27}{2} \frac{S^{7 / 3} N(S, Q, \Lambda)}{\left(3 S^{4 / 3}-Q^{2}-\Lambda S^{2}\right)^{3}\left(3 S^{4 / 3}-5 Q^{2}+\Lambda S^{2}\right)^{2}},
$$



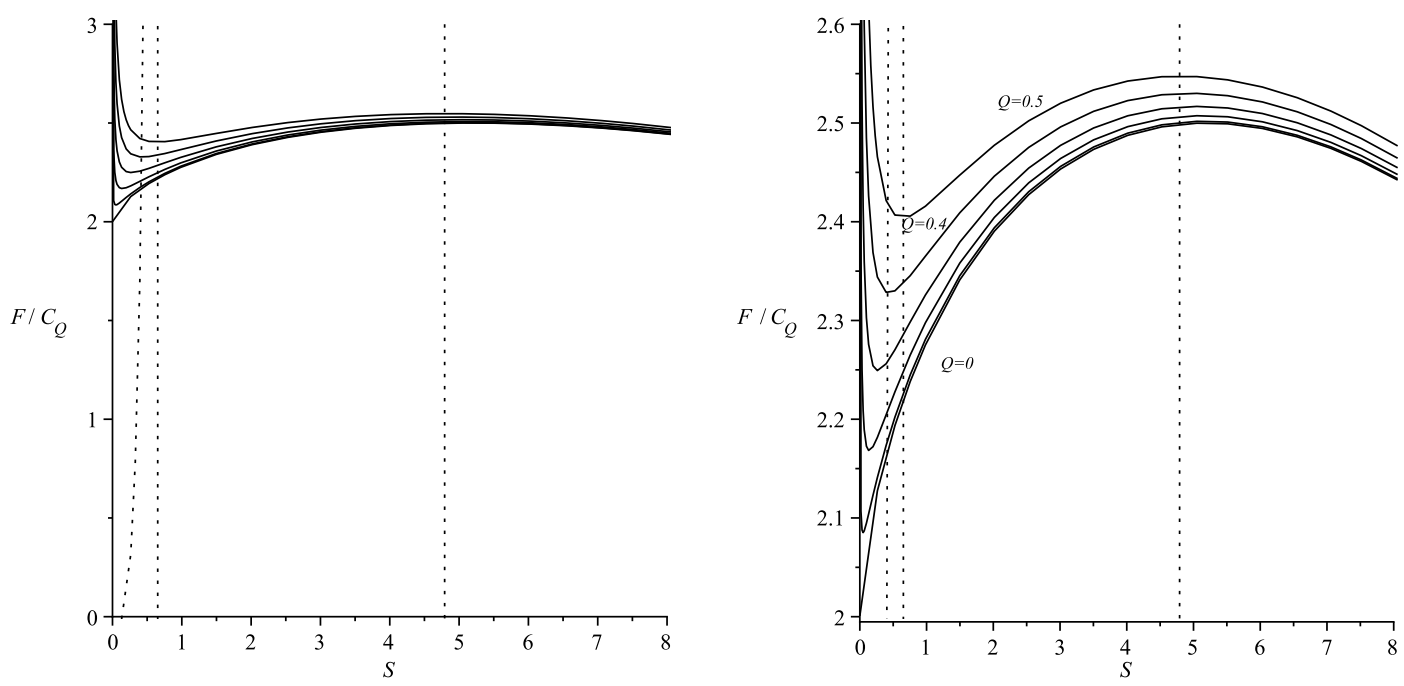

FIG. 10: The Helmholtz free energy as a function of the entropy for different values of the electric charge. The heat capacity $C_{Q}$ (dotted curve) is also depicted for $Q=0.5$ to illustrate that phase transitions occur on the minimum and maximum of $F$ for the same value of $Q$. The left plot covers a larger interval of the vertical axis to illustrate the behavior of $C_{Q}$. The right plot illustrates in more detail the behavior of $F$.

with

$$
\begin{aligned}
N(S, Q, \Lambda)= & 42 Q^{2} S^{7 / 3} \Lambda-34 S Q^{4} \Lambda-5 S^{3} Q^{2} \Lambda^{2}-18 Q^{4} \sqrt[3]{S} \\
& -7 S^{5} \Lambda^{3}+36 S^{11 / 3} \Lambda+15 S^{13 / 3} \Lambda^{2}-162 S^{3}+108 Q^{2} S^{5 / 3}
\end{aligned}
$$

From the expression for the scalar curvature it is obvious that the singularities are located at the points satisfying the equation $3 S^{4 / 3}-Q^{2}-\Lambda S^{2}=0$, which coincide with the points where $T \rightarrow 0$, and at the points satisfying the equation $3 S^{4 / 3}-5 Q^{2}+\Lambda S^{2}=0$, which are the points where $C_{Q} \rightarrow \infty$. For instance, for the particular case $\Lambda=-1$ and $Q=1 / 2$ the singularities are shown in Fig,11, their locations clearly coincide with the points where second order phase transitions occur (see right plot in Fig (6).

To see if the phase transitions predicted by $C_{\phi}$ can also be described in the context of GTD, let us consider the thermodynamic metric (3) with the the canonical. In this case, the thermodynamic potential is $\Phi=H$ and $E^{a}=(S, \phi)$ are the coordinates of the equilibrium manifold. Then, using the fundamental equation (47), we obtain from (3) the metric

$$
g=\left(1-\frac{3}{4} \phi^{2}-\frac{\Lambda}{3} S^{2 / 3}\right)\left[\frac{4}{27} S^{-2 / 3}\left(1-\frac{3}{4} \phi^{2}+\frac{\Lambda}{3} S^{2 / 3}\right) d S^{2}-S^{4 / 3} d \phi^{2}\right],
$$




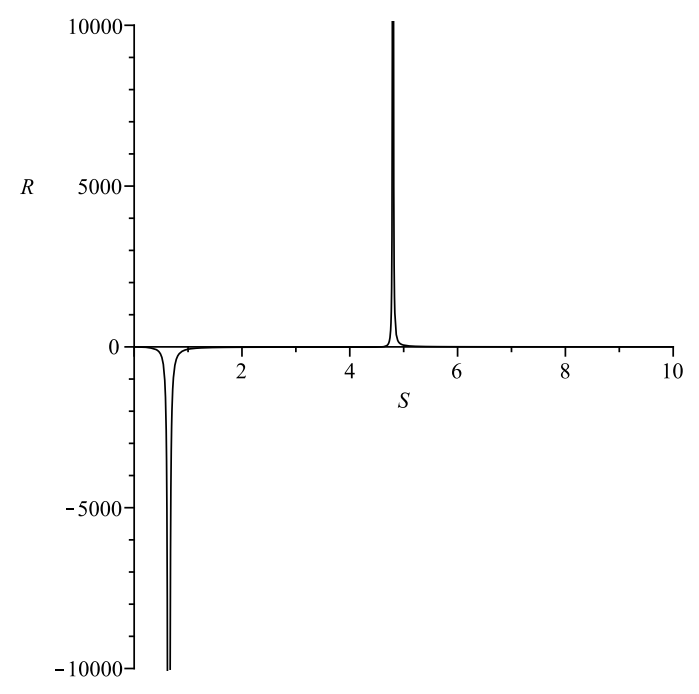

FIG. 11: Curvature singularities in the equilibrium manifold of the black hole (40) with $\Lambda=-1$ and $Q=1 / 2$. The singularities are located at $S \approx 0.6$ and $S \approx 4.9$.

and the corresponding curvature scalar

$$
R=\frac{N(S, \phi, \Lambda)}{\left(12 S^{1 / 3}-9 \phi^{2} S^{1 / 3}-4 \Lambda S\right)^{3}\left(12 S^{1 / 3}-9 \phi^{2} S^{1 / 3}+4 \Lambda S\right)^{2}},
$$

with

$N(S, \phi, \Lambda)=\left(12 S^{1 / 3}-9 \phi^{2} S^{1 / 3}-4 \Lambda S\right)^{2}+\Lambda S^{4 / 3}\left[27 \phi^{2}\left(4-3 \phi^{2}\right)+2 \Lambda S^{2 / 3}\left(9 \phi^{2}-4 \Lambda S^{2 / 3}-4\right)\right]$.

The curvature singularities situated at the roots of $12 S^{1 / 3}-9 \phi^{2} S^{1 / 3}+4 \Lambda S=0$ determine the phase transition structure of the black hole, because they coincide with the singularities of $C_{\phi}$. The second set of singularities for which $12 S^{1 / 3}-9 \phi^{2} S^{1 / 3}-4 \Lambda S=0$ corresponds to the limit $T \rightarrow 0$ and indicates the break down of the thermodynamic description of the black hole.

\section{B. Geometrothermodynamics with a modified entropy relation}

The modified entropy relation (31), with $k=+1$, cannot be solved in this case to obtain an explicit fundamental equation $M=M(Q, S)$. We must therefore consider the implicit fundamental equation determined by the relationships

$$
S=r_{H}^{3}+6 \tilde{\alpha} r_{H}, \quad M=\frac{\tilde{\alpha}}{3}+\frac{Q^{2}}{3 r_{H}^{2}}+r_{H}^{2}-\frac{\Lambda}{6} r_{H}^{4} .
$$


Then, the main thermodynamic variables can then be expressed as

$$
\begin{gathered}
T=\frac{2\left(3 r_{H}^{4}-Q^{2}-\Lambda r_{H}^{6}\right)}{9 r_{H}^{3}\left(r_{H}^{2}+2 \tilde{\alpha}\right)}, \\
\phi=\frac{2 Q}{3 r_{H}^{2}}, \\
C_{Q}=\frac{3 r_{H}\left(r_{H}^{2}+2 \tilde{\alpha}\right)^{2}\left(3 r_{H}^{4}-Q^{2}-\Lambda r_{H}^{6}\right)}{6 \tilde{\alpha} Q^{2}+5 Q^{2} r_{H}^{2}+6 \tilde{\alpha} r_{H}^{4}-3 r_{H}^{6}(1+2 \tilde{\alpha} \Lambda)-\Lambda r_{H}^{8}} .
\end{gathered}
$$

For a physically reasonable configuration we demand the positiveness of the temperature; this implies that $3 r_{H}^{4}-\Lambda r_{H}^{6}>Q^{2}$. For a given value of $\Lambda$ and $Q$, this condition determines a minimum horizon radius $r_{H}^{m i n}$ for which the temperature is positive. Moreover, from the expression for the heat capacity (59) and from the condition of positive temperature, it follows that if the condition

$$
\tilde{\alpha}|\Lambda| \geq \frac{1}{2}
$$

is satisfied, the heat capacity is positive and, consequently, all possible black hole configurations are stable. This is an interesting condition that relates two fundamental constants, namely, the tension of the string, proportional to $\tilde{\alpha}^{-1}$, and the cosmological constant $\Lambda$.

For the range $\tilde{\alpha}|\Lambda|<1 / 2$ where unstable states in principle can exist, let us consider the parameters $\Lambda=-1$ and $Q=1$. This choice together with the positiveness condition of the temperature fix the value of $r_{H}^{\min } \approx 0.73$ (see left plot in Fig, 12). Notice that the value of $r_{H}^{\min }$ does not depend on the value of the coupling constant $\tilde{\alpha}$. We explore the behavior of the heat capacity in Fig 12 for the entire range $\tilde{\alpha} \in(0,1 / 2)$, according to the condition $\tilde{\alpha}|\Lambda|<1 / 2$. One can see that the heat capacity is represented by a smooth positive function in the entire domain. We conclude that also in this case all the black hole configurations are stable.

We now investigate the geometric properties of the equilibrium manifold. According to the implicit fundamental equation, the thermodynamic metric (3) can be written as

$g=-f_{1}\left(r_{H}, Q, \tilde{\alpha}, \Lambda\right)\left\{\left(5 Q^{2} r_{H}^{2}+6 \tilde{\alpha} r_{H}^{4}-3 r_{H}^{6}(1+2 \tilde{\alpha} \Lambda)-\Lambda r_{H}^{8}\right) d S^{2}+9 r_{H}\left(r_{H}^{2}+2 \tilde{\alpha}\right)^{2} d Q^{2}\right\}$,

where

$$
f_{1}\left(r_{H}, Q, \tilde{\alpha}, \Lambda\right)=\frac{4}{243} \frac{\left(r_{H}^{2}+6 \tilde{\alpha}\right)\left(3 r_{H}^{4}-Q^{2}-\Lambda r_{H}^{6}\right)}{r_{H}^{6}\left(r_{H}^{2}+2 \tilde{\alpha}\right)^{4}} .
$$



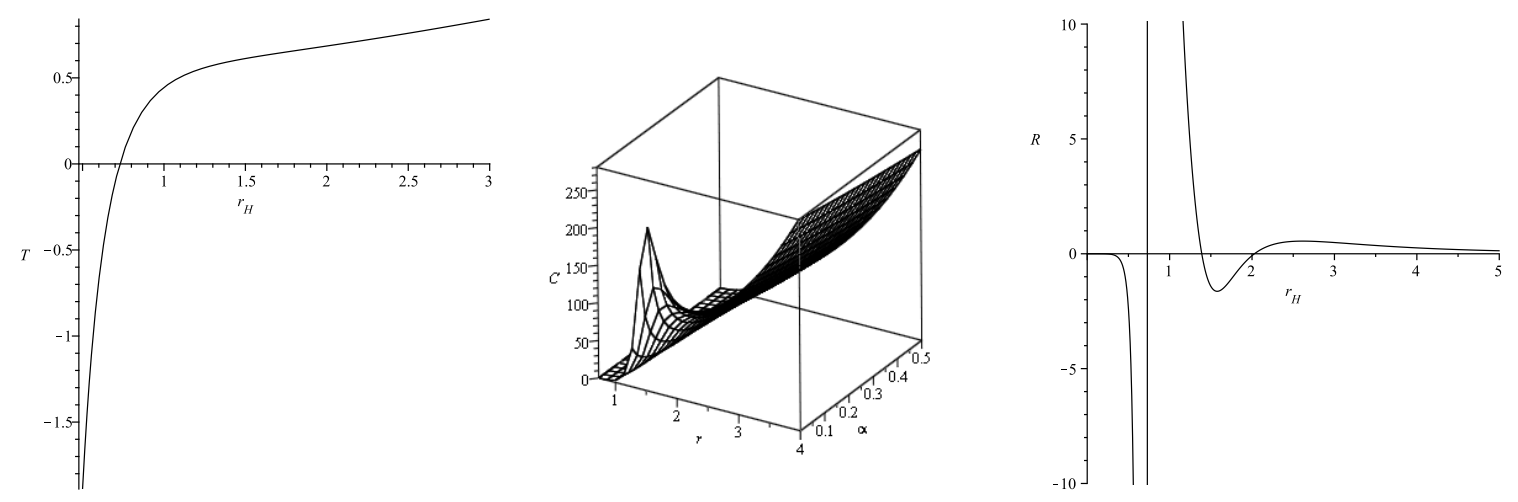

FIG. 12: Temperature, heat capacity $C_{Q}$, and thermodynamic curvature for a black hole of the EMGB theory with cosmological constant $\Lambda=-1$, charge $Q=1$, and coupling constant $\tilde{\alpha}=1 / 4$ (for the temperature) and $\tilde{\alpha} \in(0,0.5)$ (for the heat capacity). The temperature is positive for $r_{H}>r_{H}^{\min } \approx 0.73$.

The expression for the scalar is quite cumbersome but it can schematically be represented as

$$
R=\frac{N\left(r_{H}, \Lambda, Q, \tilde{\alpha}\right)}{\left(3 r_{H}^{4}-Q^{2}-\Lambda r_{H}^{6}\right)^{3}\left(6 \tilde{\alpha} Q^{2}+5 Q^{2} r_{H}^{2}+6 \tilde{\alpha} r_{H}^{4}-3 r_{H}^{6}(1+2 \tilde{\alpha} \Lambda)-\Lambda r_{H}^{8}\right)^{2}\left(r_{H}^{2}+6 \tilde{\alpha}\right)^{3}},
$$

where $N\left(r_{H}, Q, \Lambda, \tilde{\alpha}\right)$ is a finite function in the entire domain of definition. From the expression for the scalar curvature, the temperature and heat capacity given above, it follows that singularities can take place only at those points where $T \rightarrow 0$ or $C \rightarrow \infty$. In Fig, 12 , the behavior of the scalar curvature is shown for a particular choice of the parameters. We see that a singularity occurs at the point where the temperature vanishes. The singularity situated at $\left(r_{H}^{2}+6 \tilde{\alpha}\right)=0$ corresponds to the limit $S \rightarrow 0$ which indicates the breakdown of the thermodynamic picture of the black hole and, hence of GTD. No other singularities exist because the heat capacity is finite in this domain.

\section{SPHERICALLY SYMMETRIC BLACK HOLE IN EYMGB GRAVITY}

In this section we first describe the black hole solution [48] and its properties in EinsteinYang-Mills-Gauss-Bonnet (EYMGB) gravity, and then study the geometry of the black hole thermodynamics in the subsequent sections.

The 5D spherically symmetric solution obtained recently by Mazharimousavi and Halisoy 
[48] has the metric

$$
d s^{2}=-f(r) d t^{2}+\frac{d r^{2}}{f(r)}+r^{2} d \Omega_{3}^{2}
$$

where

$$
f(r)=1+\frac{r^{2}}{4 \alpha}-\sqrt{1+\frac{M}{2 \alpha}+\frac{r^{4}}{16 \alpha^{2}}+\frac{Q^{2}}{\alpha} \ln r} .
$$

Here $M$ is an integration constant to be identified as the mass, and $Q$ is the only non-zero gauge charge. The Yang-Mills field 2-form $F_{\alpha \beta}^{i} F^{i \alpha \beta}=6 Q^{2} / r^{4}$ represents in this case the matter Lagrangian in the general action (5). The metric on the unit three sphere $d \Omega_{3}^{2}$ is given by

$$
d \Omega_{3}^{2}=\frac{1}{4}\left(d \theta^{2}+d \phi^{2}+d \psi^{2}-2 \cos \theta d \phi d \psi\right),
$$

with $\theta \in[0, \pi]$, and $(\phi, \psi) \in[0,2 \pi]$. The event horizon radius $r_{H}$ satisfies the equation $f\left(r_{H}\right)=0$ and is given by

$$
M=r_{H}^{2}-2 Q^{2} \ln r_{H} .
$$

This black hole solution of the EYMGB theory is well defined for all $r$ if the GaussBonnet coupling parameter $\alpha$ is positive definite. For $\alpha<0$, the spacetime has a curvature singularity at the hypersurface $r=r_{s}$, where $r_{s}$ is the largest root of $f(r)=0$.

\section{A. Analysis with the Bekenstein-Hawking entropy relation}

In suitable units, the entropy $S$ of the black hole is given by $S=r_{H}^{3}$, and $A=2 \pi^{2} r_{H}^{3}$ is the surface area of the event horizon. Then, according to Eq.(67), the thermodynamic fundamental equation in the $M$-representation becomes

$$
M=S^{\frac{2}{3}}-\frac{2}{3} Q^{2} \ln S
$$

This is the main relationship from which all the thermodynamic properties of this black hole can be derived.

\section{Thermodynamics}

The expressions for the main thermodynamic quantities, namely, the temperature and the electric potential are given by

$$
T=\frac{2}{3} \frac{S^{2 / 3}-Q^{2}}{S}, \quad \phi=-\frac{4}{3} Q \ln S .
$$



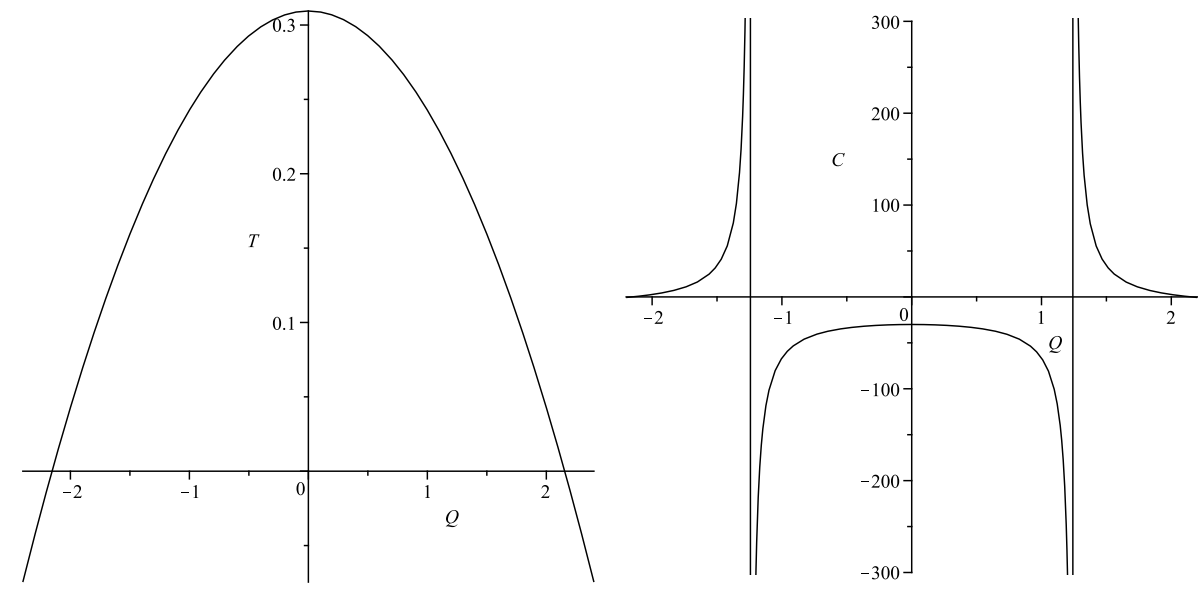

FIG. 13: Temperature and the heat capacity $C_{Q}$ in terms of the Yang-Mills charge $Q$. To illustrate the thermodynamic behavior, the Bekenstein-Hawking entropy was chosen as $S=10$.

It follows that for the temperature of the black hole to be positive the charge must satisfy the condition $Q<S^{1 / 3}$. Moreover, for a fixed value of the entropy, the maximum temperature is reached at the value $Q=0$, indicating that the Yang-Mills charge reduces the temperature of the black hole. This behavior is illustrated in Fig,13.

Now, in the grand canonical ensemble the heat capacity is given by the expression

$$
C_{Q}=-3 S \frac{S^{2 / 3}-Q^{2}}{S^{2 / 3}-3 Q^{2}} .
$$

In the region $Q<S^{1 / 3}$, where the temperature is positive, the heat capacity diverges at those points where $Q=S^{1 / 3} / \sqrt{3}$, indicating the existence of a second order phase transition. In the interval $S^{1 / 3} / \sqrt{3}<|Q|<S^{1 / 3}$, the heat capacity is positive (and $T>0$ ), i. e., the black hole configuration is stable in this interval. Furthermore, the heat capacity is negative within the interval $0<|Q|<S^{1 / 3} / \sqrt{3}$ which corresponds to an unstable thermodynamic configuration. Since the heat capacity at $Q=0$ is negative, we conclude that the addition of a Yang-Mills charge $Q$ to an unstable neutral black hole not only reduces its temperature, but also changes its heat capacity until it becomes positive and the system becomes stable, if the charge is sufficiently large. The transition from an unstable state to a stable state is accompanied by a second order phase transition. This thermodynamic behavior is illustrated in Fig.13.

Consider now the canonical ensemble that is determined by the thermodynamic potential

$$
H \equiv M-\phi Q=S^{2 / 3}+\frac{3}{8} \frac{\phi^{2}}{\ln S}
$$



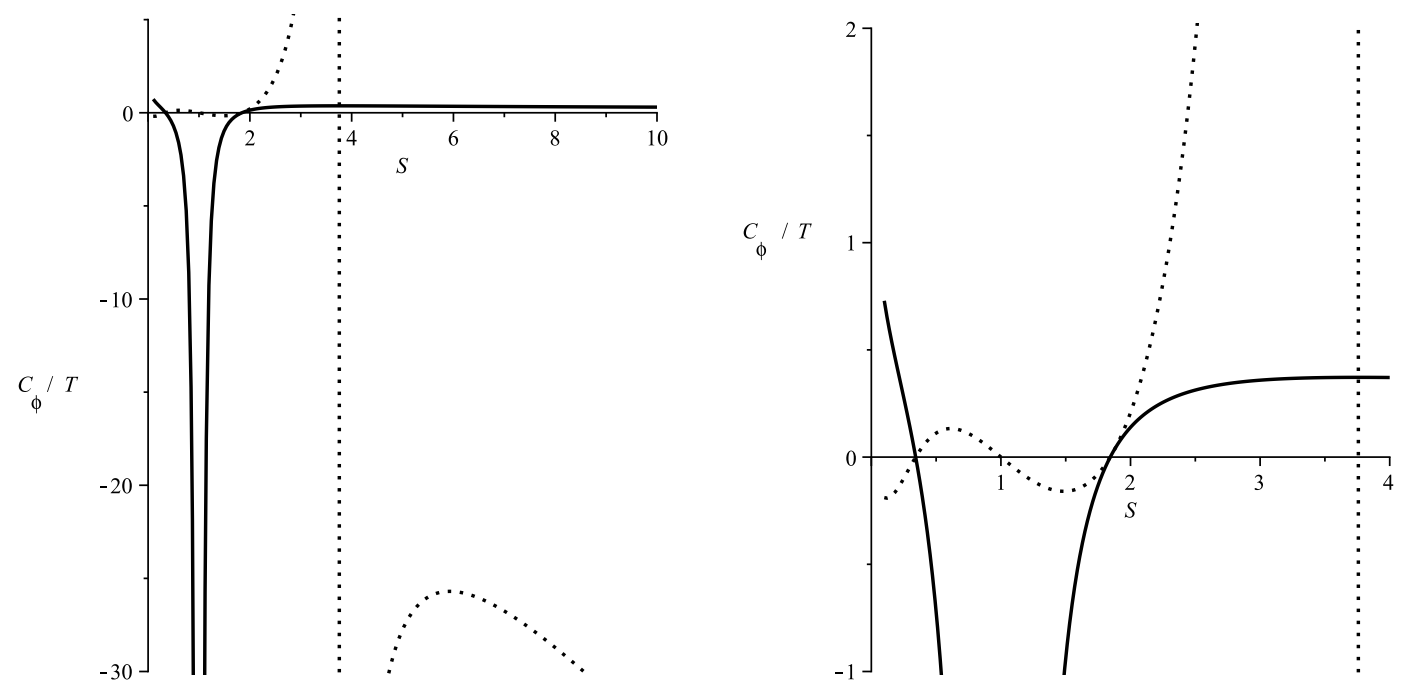

FIG. 14: The heat capacity $C_{\phi}$ (dotted curve) and the temperature (solid curve) in terms of the entropy. Here $\phi=1$ and $\Lambda=-1$.

from which the dual thermodynamic variables are obtained as

$$
T=\frac{16 S^{2 / 3} \ln ^{2} S-9 \phi^{2}}{24 S \ln ^{2} S}, \quad Q=-\frac{3 \phi}{4 \ln S} .
$$

Furthermore, the heat capacity at fixed potential is given by

$$
C_{\phi}=\left(\frac{\partial H}{\partial T}\right)_{\phi}=\frac{3 S \ln S\left(16 S^{2 / 3} \ln ^{2} S-9 \phi^{2}\right)}{-16 S^{2 / 3} \ln ^{3} S+27 \phi^{2}(2+\ln S)},
$$

and predicts a phase structure different from that of $C_{Q}$ as given in Eq.(70). Indeed, in Fig. 14 we illustrate the behavior of $C_{\phi}$ and the temperature as functions of the entropy. In the interval $S \in(0,0.4)$ with positive temperature, the black hole is unstable. Then, in the interval $S \in(0.4,1.8)$ no black holes can exist with positive temperature. In the following interval $S \in(1.8,3.7)$ all black holes are stable and have positive temperature. The vertical dotted line situated at $S \approx 3.7$ denotes the singularity of $C_{\phi}$ that corresponds to a second order phase transition during which the stable black hole changes into an unstable state.

To investigate the position of the phase transitions in the parameter space we consider all the thermodynamic potentials $M, H, F$, and $G$. Their behavior is depicted in Fig. 15. It can be seen that at the point where $C_{\phi}$ diverges, all the potentials are well-behaved with no extrema. As for the heat capacity $C_{Q}$, the location of the singularity coincides with the only minimum of $F$. In Fig. 16, the behavior of $F$ and $C_{Q}$ are depicted for different values of the charge. It follows that the phase transitions take place in regions where the Helmholtz 

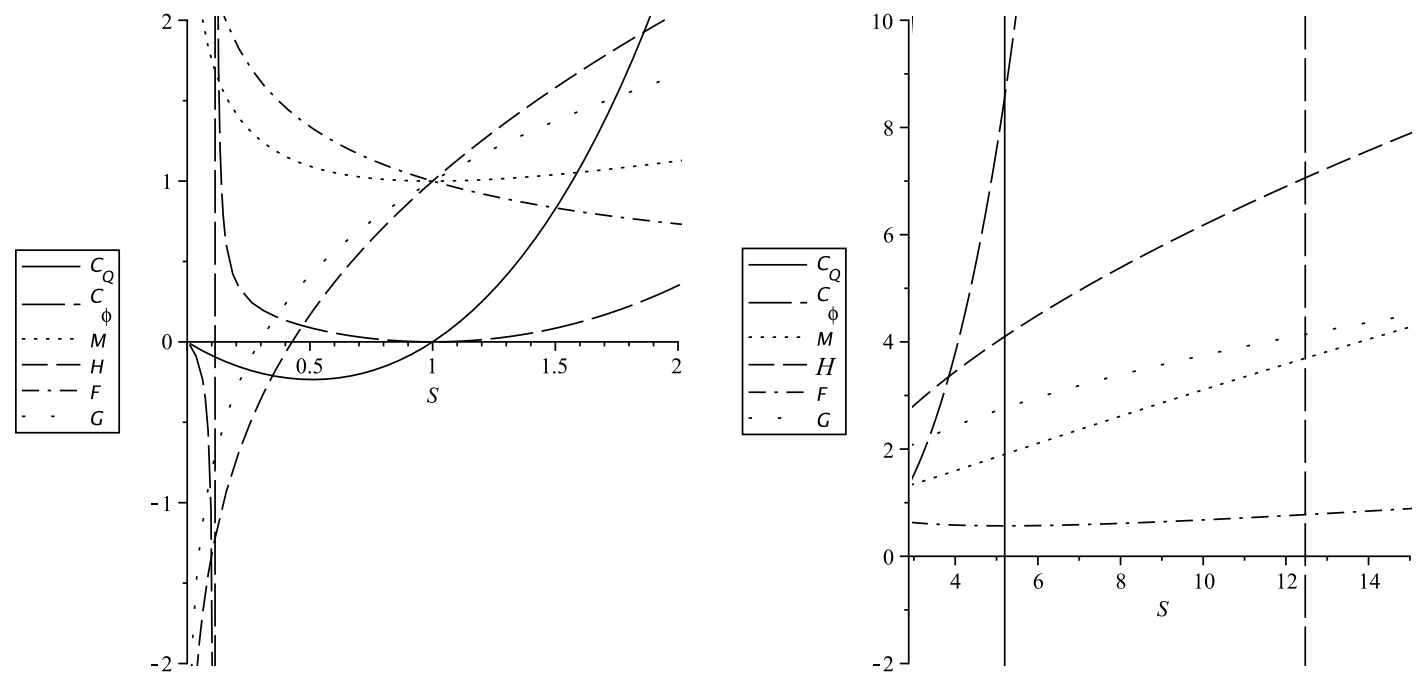

FIG. 15: Heat capacities and thermodynamic potentials of the EYMGB black hole for the particular value $Q=1$.

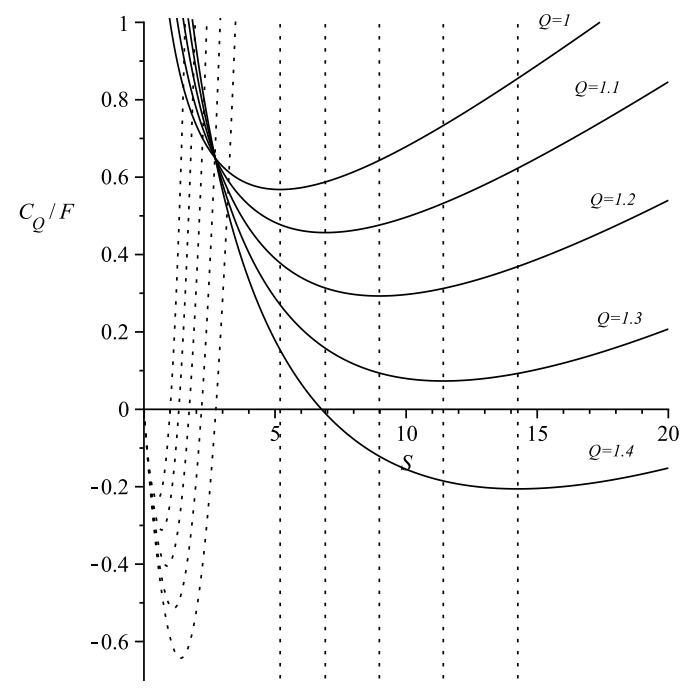

FIG. 16: The Helmholtz free energy $F$ (solid curves) and the heat capacity $C_{Q}$ (dotted durves) in terms of the entropy for different values of the entropy. In each case, the minimum of $F$ coincides with the divergence of $C_{Q}$.

potential is stable. 


\section{Geometrothermodynamics}

To investigate the geometry of the corresponding equilibrium manifold in the grand canonical ensemble, we use the general metric (3) with $\Phi=M$ and $E^{a}=(S, Q)$, and the fundamental equation $M=M(S, Q)$ as given in Eq.(68). Then, we obtain

$$
g=\frac{4}{27}\left(S^{2 / 3}-Q^{2}\right)\left(\frac{S^{2 / 3}-3 Q^{2}}{S^{2}} d S^{2}-6 \ln S d Q^{2}\right) .
$$

The corresponding thermodynamic curvature scalar can be represented as

$$
R=\frac{N(S, Q)}{\left(S^{2 / 3}-Q^{2}\right)^{3}\left(S^{2 / 3}-3 Q^{2}\right)^{2} \ln ^{2} S},
$$

where $N(S, Q)$ is a well-behaved function of its arguments. We see that there are several places where true curvature singularities can exist. First, if $Q=S^{1 / 3}$ the curvature scalar diverges and, as described above, the temperature vanishes. Then, at $Q=S^{1 / 3} / \sqrt{3}$ there exists a singularity whose location coincides with the values at which the heat capacity $C_{Q}$ diverges and second order phase transitions occur. Finally, if $\ln S \rightarrow 0$ the curvature scalar diverges. We interpret this additional singularity as related to a second order phase transition which is not contained in $C_{Q}$. In fact, in analogy to the heat capacity $C_{Q}$ defined in Eq.(70), we can introduce the capacitance $C_{S} \equiv(\partial Q / \partial \Phi)_{S}=(\partial \Phi / \partial Q)_{S}^{-1}=\left(\partial^{2} M / \partial Q^{2}\right)_{S}^{-1}$. Then, from the fundamental equation (68), we obtain $C_{S}=-3 /(4 \ln S)$ so that in the limit $S \rightarrow 1$ a second order phase transition occurs. This proves the physical origin of the additional singularity of the thermodynamic curvature. The behavior of this thermodynamic curvature is depicted in Fig 17 .

We now investigate the geometric properties of the equilibrium manifold of this black hole in the canonical ensemble. The fundamental equation $H=H(S, \phi)$ is given by Eq.(71) so that we can identify the thermodynamic potential as $\Phi=H$ and $E^{a}=(S, \phi)$. Then, from the general thermodynamic metric (3) we obtain

$$
g=\frac{16 S^{2 / 3} \ln ^{2} S-9 \phi^{2}}{\ln ^{3} S}\left[\frac{-16 S^{2 / 3} \ln ^{3} S+27 \phi^{2}(2+\ln S)}{54 S^{2} \ln ^{2} S} d S^{2}-d \phi^{2}\right],
$$

whose curvature scalar can be represented as

$$
R=\frac{N(S, \phi)}{\left(-16 S \ln ^{3} S+54 \phi^{2} S^{1 / 3}+27 \phi^{2} S^{1 / 3} \ln S\right)^{2}\left(16 S \ln ^{2} S-9 \phi^{2} S^{1 / 3}\right)^{3}} .
$$

The first term in the denominator of $R$ coincides with the denominator of $C_{\phi}$ and, conse-

quently, reproduces exactly the phase transition structure of the black hole. The second term 


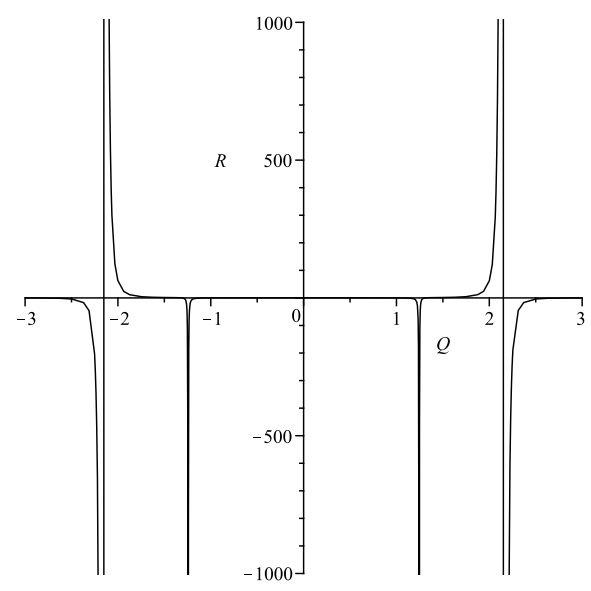

FIG. 17: The thermodynamic curvature with $S=10$ as a function of the Yang-Mills charge $Q$. Singularities are due to the existence of a second order phase transition at $Q \approx \pm 1.24$ or to the vanishing of the temperature at $Q \approx \pm 2.15$.

in the denominator of $R$ vanishes as $T \rightarrow 0$, indicating the break down of the thermodynamic and geometrothermodynamic descriptions.

\section{B. Geometrothermodynamics with a modified entropy relation}

In this case, the thermodynamic fundamental equation cannot be written explicitly. Therefore, we use the implicit equation $S=S(M, Q)$ determined by the expressions

$$
S=r_{H}^{3}+6 \tilde{\alpha} r_{H}, \quad \text { and } \quad M=r_{H}^{2}-2 Q^{2} \ln r_{H}
$$

Then, we find the following thermodynamic variables

$$
\begin{gathered}
T=\frac{2}{3} \frac{r_{H}^{2}-Q^{2}}{r_{H}\left(r_{H}^{2}+2 \tilde{\alpha}\right)}, \\
\phi=4 Q \ln r_{H} \\
C_{Q}=\frac{3 r_{H}\left(r_{H}^{2}-Q^{2}\right)\left(r_{H}^{2}+2 \tilde{\alpha}\right)^{2}}{-r_{H}^{4}+\left(2 \tilde{\alpha}+3 Q^{2}\right) r_{H}^{2}+2 Q^{2} \tilde{\alpha}} .
\end{gathered}
$$

Notice that in this case the condition for a positive definite temperature reads $r_{H}^{2}>Q^{2}$. Moreover, the explicit presence of the coupling constant $\tilde{\alpha}$ in the heat capacity leads to the possibility of modifying the phase transition structure of the black hole by changing the value of the GB coupling constant. Indeed, the expression for the heat capacity (81) 

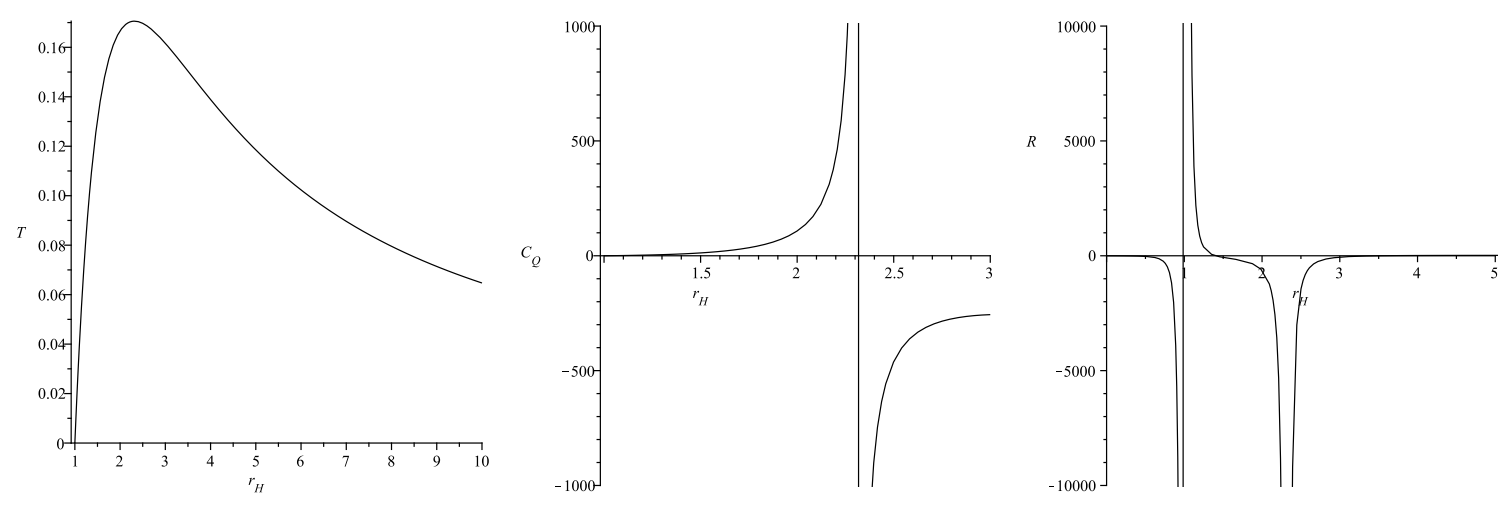

FIG. 18: Temperature, heat capacity, and scalar curvature in terms of the horizon radius $r_{H}$ of a black in the EYMGB theory. Here the modified entropy relation is used with $Q=1$ and $\tilde{\alpha}=1$.

diverges for

$$
r_{H}^{2}=\frac{3}{2} Q^{2}+\tilde{\alpha} \pm \frac{1}{2} \sqrt{9 Q^{4}+20 \tilde{\alpha} Q^{2}+4 \tilde{\alpha}^{2}},
$$

indicating that for a given value of the Yang-Mills charge it is possible to find a range of values of $\tilde{\alpha}$ for which second order phase transitions take place. This behavior is illustrated in Fig 18

For the thermodynamic system determined by the fundamental equation (78), the Legendre invariant metric (3) is given by

$g=-\frac{4}{27} \frac{\left(r_{H}^{2}+6 \tilde{\alpha}\right)\left(r_{H}^{2}-Q^{2}\right)}{r_{H}^{2}\left(r_{H}^{2}+2 \tilde{\alpha}\right)^{4}}\left\{\left[-r_{H}^{4}+\left(2 \tilde{\alpha}+3 Q^{2}\right) r_{H}^{2}+2 Q^{2} \tilde{\alpha}\right] d S^{2}+18 r_{H}^{2}\left(r_{H}^{2}+2 \tilde{\alpha}\right)^{3} \ln r_{H} d Q^{2}\right\}$,

The expression for the scalar curvature can be schematically written as

$$
R=\frac{N\left(r_{H}, Q, \tilde{\alpha}\right)}{\left[-r_{H}^{4}+\left(2 \tilde{\alpha}+3 Q^{2}\right) r_{H}^{2}+2 Q^{2} \tilde{\alpha}\right]^{2}\left(r_{H}^{2}-Q^{2}\right)^{3}\left(r_{H}^{2}+6 \tilde{\alpha}\right)^{3}\left(\ln r_{H}\right)^{2}},
$$

where $N\left(r_{H}, Q, \tilde{\alpha}\right)$ is a function that is finite at those points where the denominator vanishes. We see that curvature singularities occur at $r_{H}^{2}=Q^{2}$, which is the point where the temperature vanishes, and at the roots of Eq.(82), which determine the points where $C_{Q} \rightarrow \infty$ and second order phase transitions occur. The singularity situated at $\ln r_{H} \rightarrow 0$ corresponds to a second order phase transition determined by the capacitance $C_{S} \equiv(\partial Q / \partial \Phi)_{S}=-1 /\left(4 \ln r_{H}\right)$, according to Eq.(78)). Finally, the singularity situated at $\left(r_{H}^{2}+6 \tilde{\alpha}\right)=0$ corresponds to the limit $S \rightarrow 0$ which indicates the breakdown of the thermodynamic picture of the black hole and, consequently, of GTD. A particular example of the location of these curvature singularities is depicted in Fig, 18, 


\section{CONCLUSIONS}

In this work, we analyzed the thermodynamics of static spherically symmetric black holes in the five dimensional Einstein-Gauss-Bonnet theory and its generalizations including an electromagnetic Maxwell field (EMGB), a cosmological constant (EMGB $\Lambda$ ), and a YangMills field (EYMGB). This kind of black holes was also recently investigated in [49] with results which are compatible with the ones obtained in the present analysis. To investigate the thermodynamics of these black holes we use two different approaches. The first one is based upon the Bekenstein-Hawking entropy relation, according to which the entropy of a black hole is proportional to the area of its event horizon. The second approach uses as starting point a modified entropy relation that follows from the assumption that black holes satisfy the first law of thermodynamics in higher dimensions. The two approaches are not equivalent since the corresponding thermodynamic variables exhibit completely different behaviors. In particular, we noticed that the thermodynamics of black holes based upon the modified entropy formula depends on the value of the coupling constant of the GaussBonnet term that appears in the action of the theory. Phase transitions appear that depend on the explicit value of the coupling constant, and change the stability properties of the black holes. This kind of phase transitions is absent when the Bekenstein-Hawking entropy relation is used.

To study the phase transition structure that follows from the Bekenstein-Hawking entropy we used the original Davies' definition, according to which divergences of the heat capacity at fixed charge $C_{Q}$ represent second order phase transitions, and an alternative approach based on the analysis of the divergences of $C_{\phi}$, where $\phi$ is the electric potential dual to $Q$. For all three black holes studied in this work, we showed that the divergences of $C_{Q}$ do not coincide with the divergences of $C_{\phi}$ and, consequently, the corresponding phase transition structures are different. Moreover, we analyzed the behavior of all possible thermodynamic potentials in the parameter space $S-Q$, and found that the divergences of $C_{\phi}$ do not correspond to any particular point in the parameter space. On the contrary, the divergences of $C_{Q}$ are always situated on points where the Helmholtz free energy $F$ possesses an extremum. The remaining thermodynamic potentials do not show any special behavior at the singular points. To be more specific, in the case of the EMGB black holes we found that $C_{Q}$ predicts the existence of stable and unstable states with a phase transition that occurs in a region 
of stability of $F$, and during which the black holes undergoes a transition from a stable to an unstable state. For this black hole, the heat capacity $C_{\phi}$ implies the existence of only unstable states with no phase transitions. In the case of the EMGB black hole with cosmological constant, $C_{Q}$ predicts two phase transitions. During the first transition the black hole goes from a stable state to an unstable one whereas at the second divergence of $C_{Q}$ the black hole undergoes a phase transition from an unstable state to a stable state. In the parameter space, the first phase transition turns out to be located in a metastable region of $F$, and the second transition is situated in an unstable region of $F$. The alternative heat capacity $C_{\phi}$ predicts stable and unstable states with two different divergences. At the first divergence, the black hole undergoes a transition from a stable state to an unstable state, and at the second divergence it becomes stable again. None of the thermodynamic potentials present extrema on the divergences of $C_{\phi}$. As for the EYMGB black hole, the corresponding heat capacity $C_{Q}$ contains a singularity that describes the transition from a stable state to an unstable one. The singularity is located on a stable point of $F$. Finally, the capacity $C_{\phi}$ predicts two divergences the first of which corresponds to a transition from an unstable to a stable state whereas the second divergence corresponds to a transition to an unstable region.

For all the black holes analyzed in this work, we use the formalism of geometrothermodynamics (GTD) to find the geometric properties of the corresponding manifolds of equilibrium states. Once the thermodynamic fundamental equation of the black is given, a standard procedure of GTD allows us to compute the explicit form of the thermodynamic metric that describes the geometric properties of the equilibrium manifold. It turns out that the thermodynamic metrics depend on the entropy relation used to construct the thermodynamics of the black holes under consideration. The thermodynamic metrics obtained from the Bekenstein-Hawking relation were derived explicitly for the grand canonical ensemble, with the thermodynamic potential $M=M(S, Q)$, and for the canonical ensemble, with potential $H=H(S, \phi)$. In the first case, the curvature of the equilibrium manifold reproduces the thermodynamic behavior of the heat capacity at fixed charge $C_{Q}$ and, in the second case, the phase structure of $C_{\phi}$ is reproduced correctly. We also investigated an alternative thermodynamics based upon the modified entropy relation. In this case, we calculated for all the black holes the heat capacity $C_{Q}$, which predicts a non-trivial phase transition structure, and showed that GTD represents correctly the phase transitions as curvature singularities. 
Moreover, curvature singularities also appear at those points where the temperature vanishes, indicating the limit of applicability of black hole thermodynamics and of GTD.

In the case of the thermodynamics based on the modified entropy relation, in principle, it could be possible to perform a similar analysis with different heat capacities and thermodynamic potentials. The computations, however, cannot be carried out in a similar manner, because it is not possible to write down explicitly the intensive variables in terms of the extensive ones. This means, for instance, that we cannot write explicitly the enthalpy $H$ in terms of $S$ and $\phi$ and so it is not possible to directly compute the thermodynamic metric, using the fundamental equation $H(S, \phi)$. Of course, one could express all the derivatives with respect to $\phi$ in terms of derivatives with respect to the radius of the horizon $r_{H}$ that, in turn, is a function of $S$ and of the implicit function $Q(\phi)$. This is not an impossible task, but the computations become rather cumbersome; we do not believe that such an analysis would shed more light on the properties of GTD that has shown already to correctly describe the thermodynamics based on the Bekenstein-Hawking entropy relation.

We conclude that the formalism of GTD can be used in the EGB theory in five dimensions to describe in an invariant manner the thermodynamic properties of black holes in terms of geometric concepts, regardless of the entropy relation used to formulate the thermodynamics of black holes. In particular, we found that all the black holes we analyzed in the EGB theory are characterized by non-flat equilibrium manifolds. This means that all those black holes possess an intrinsic non-trivial thermodynamic interaction. Moreover, since we represent the thermodynamic interaction by means of the curvature of the equilibrium manifold, the points where the heat capacity diverges and, consequently, second order phase transitions occur, are represented in GTD by curvature singularities, indicating the limit of applicability of the thermodynamic approach to black holes and of GTD.

We have seen that the phase transition structure of EMG black holes depend upon the thermodynamic ensemble, because the corresponding heat capacities have different singular behaviors. It is not clear which structure is the right one. Moreover, the investigation of thermodynamic potentials does not seem to clarify this question, because no obvious relationship was found between the critical points of the potentials and the divergences of the heat capacities. Although we found that the divergences of the heat capacity at fixed charge always occur at points where the Helmholtz free energy has extrema, it is not clear why we should not take into account divergences of heat capacities that are not related to 
extrema of the potentials. We believe that a classification of phase transitions that would take into account the Legendre invariance of ordinary thermodynamics could shed some light into this issue. We expect to consider this problem in the near future.

\section{Acknowledgements}

The authors are grateful to ICRANet for warm hospitality and support. This work was supported in part by DGAPA-UNAM, grant No. IN106110, and Conacyt-Mexico, grant No. 166391.

[1] P. C. W. Davies, Proc. Roy. Soc. Lond. A 353499 (1977); P. C. W. Davies, Rep. Prog. Phys. 41 1313(1977); P. C. W. Davies, Class. Quant. Grav. 61909 (1989).

[2] R. M. Wald, Living Rev. Rel. 4, 6 (2001).

[3] J. D. Bekenstein, Phys. Rev. D 72333 (1973).

[4] S. W. Hawking, Commun. Math. Phys. 43199 (1975).

[5] J. M. Bardeen, B. Carter, and S. W. Hawking, Commun. Math. Phys. 31161 (1973).

[6] F. Weinhold, J. Chem. Phys. 632479 (1975).

[7] G. Ruppeiner, Phys. Rev. A 201608 (1979).

[8] P. Salamon, E. Ihrig, and R. S. Berry, J. Math. Phys. 242515 (1983); R. Mrugala, J. D. Nulton, J. C. Schon, and P. Salamon, Phys. Rev. A 413156 (1990).

[9] H. B. Callen, Thermodynamics and an introduction to thermostatics (John Wiley \& Sons, Inc., New York, 1985).

[10] J. Shen, R. Cai, B. Wang, and R. Su, Int J. Mod. Phys. A 2211 (2007); B. Mirza and M. Zamaninasab, JHEP 0706059 (2007); A. J. M. Medved, Mod. Phys. Lett. A 232149 (2008).

[11] J. E. Åman, I. Bengtsson, and N. Pidokrajt, Gen. Rel. Grav. 381305 (2006); T. Sarkar, G. Sengupta, and B. N. Tiwari, JHEP 0810076 (2008); R. Biswas and S. Chakrobarty, Gen. Rel. Grav. 4341 (2011); S. W. Wei, Y. X. Liu, Y. Q. Wang, and H. Guo, arXiv:hep-th/1002.1550

[12] R. Biswas and S. Chakraborty, Class. Quantum Grav. 25245015 (2008); R. Biswas and S. Chakraborty, Gen. Rel. Grav. 421311 (2010); R. Biswas and S. Chakraborty, Astrophys. Space Sci. 32639 (2010). 
[13] H. Quevedo, J. Math. Phys. 48013506 (2007).

[14] V. I. Arnold, Mathematical methods of classical mechanics (Springer Verlag, New York, 1980).

[15] H. Quevedo, A. Sánchez, S. Taj, and A. Vázquez, Gen. Rel. Grav. 431153 (2011).

[16] A. Vázquez, H. Quevedo, and A. Sánchez, J. Geom. Phys. 60, 1942 (2010).

[17] H. Quevedo and A. Sánchez, Phys. Rev. D 79024012 (2009).

[18] H. Quevedo, Gen. Rel. Grav. 40971 (2008).

[19] H. Quevedo and A. Vázquez, AIP Conf. Proc. 977165 (2008).

[20] J. L. Álvarez, H. Quevedo, and A. Sánchez, Phys. Rev. D 77084004 (2008).

[21] H. Quevedo and A. Sánchez, J. High Energy Phys. 09034 (2008).

[22] H. Quevedo and A. Sánchez, Phys. Rev. D 79024012 (2009).

[23] M. E. Rodrigues and Z. A. A. Oporto, (2012) arXiv:1201.5337.

[24] H. Quevedo, S. Taj, A. Sánchez and A. Vázquez, J. Korean Phys. Soc. 57646 (2011).

[25] H. Quevedo, A. Sánchez and A. Vázquez, Invariant geometry of the ideal gas, arXiv: $0811.0222[$ math-phys]

[26] L. Zhao, Commun. Theor. Phys. 561052 (2011).

[27] H. Quevedo and M. N. Quevedo, Fundamentals of Geometrothermodynamics, Elect. J. Theor. Phys. (2011).

[28] H. Quevedo, S. Taj, A. Sánchez and A. Vázquez, Geometrothermodynamics in Hořava-Lifshitz gravity, J. Phys. A (2012), in press.

[29] D. G. Boulware and S. Deser, Phys. Rev. Lett. 552656 (1985).

[30] D. Lovelock, J. Math. Phys. 12498 (1971).

[31] Sami M and Dadhich, TSPU Vestnik 44N7 25 (2004); (arXiv: hep-th/0405016r2).

[32] B. Zwiebach, Phys. Lett. B 156315 (1985); R.I. Nepomechie, Phys. Rev. D 323201 (1985).

[33] A.H. Chamseddine, Phys. Lett. B 233291 (1989); F. Muller-Hoissen, Nucl. Phys. B 349235 1990.

[34] C. Lanczos, Z. Phys. 73 (1932) 147 C. Lanczos, Z. Phys. 73147 (1932); C. Lanczos, Ann. Math. 39842 (1938); J. T. Wheeler, Nucl. Phys. B 268737 (1986); J. T. Wheeler, Nucl. Phys. B 273732 (1986); S. Nojiri and S. Odintsov, Phys. Rev. D 65023521 (2001); S. Nojiri, S. Odintsov, and S. Ogushi, Int. J. Mod. Phys. A 174809 (2002); S. Deser and B. Tekin, Phys. Rev. D 67084009 (2003); R. C. Myers and J. Z. Simon, Phys. Rev. D 382434 (1988); M. H. Dehghani and R. B. Mann, Phys. Rev. D 72124006 (2005); H. Maeda and N. Dadhich, 
Phys. Rev. D74 021501 (2006); H. Maeda and N. Dadhich, Phys. Rev. D75 044007 (2007); G. Kofinas and R. Olea, JHEP 11069 (2007); M. Thibeault, C. Simeone and E. F. Eirod, Gen. Rel. Grav. 381593 (2006); G. Kofinas and R. Olea, Phys. Rev. D 74084035 (2006).

[35] S. Chakraborty and T. Bandyopadhyay, Class. Quant. Grav. 25245015 (2008).

[36] R. C. Myers and J. Z. Simon, Phys. Rev.D 382434 (1988); R. G. Cai and Q. Guo Phys. Rev.D 69104025 (2004).

[37] S. Nojiri and S. Odintsov, Phys. Lett. B 52187 (2001).

[38] R. G. Cai, Phys. Rev. D 65084014 (2002).

[39] M. H. Dehghani, Phys. Rev. D 70064019 (2004).

[40] H. Falcke and F.W. Hehl, The Galactic Black Hole: Lectures on General Relativity and Astrophysics (Bristol: Institute of Physics Publishing, 2003)

[41] P. C. W. Davies, Rep. Prog. Phys. 411313 (1978).

[42] Y.-H. Wei, Phys. Rev. D 80024029 (2009).

[43] Y.S. Myung, Y.- W. Kim and Y.-J. Park, Phys. Lett. B 663342 (2008).

[44] R. Cai and K. Soh, Phys. Rev. D 59044013 (1999).

[45] M. Cvetic, S. Nojiri and S. Odintsov, Nucl. Phys. B 628295 (2002).

[46] T. Clunan, S. F. Ross and D. J. Smith, Class. Quant. Grav. 21 3447(2004).

[47] D. L. Wiltshire, Phys. Lett. B 16936 (1986); D. L. Wiltshire, Phys. Rev. D 382445 (1988).

[48] S. H. Mazharimousavi and M. Halisoy, Phys. Rev.D 76087501 (2007).

[49] R. Chowdhury, R. Biswas, N. Mazumder, and S. Chakraborty, Int. J. Theor. Phys. 501628 (2011).

[50] This simple observation turns out to be the starting point to formulate an invariant classification of phase transitions (H. Quevedo, An invariant classification of phase transitions (2012), in preparation) which can be used, in particular, to clarify certain puzzling results found recently in black hole phase transitions by N. Pidokrajt, and J. Ward, arXiv:gr-qc/1106.2831.

[51] It is widely believed that to solve this problem it is necessary to start from a consistent quantum theory of gravity that is still unknown.

[52] Using the "heat" as starting point, one can only introduce $C_{Q}$ and $C_{\phi}$. However, since these capacities can also be expressed as $C_{X}=\left(T \frac{\partial S}{\partial T}\right)_{X}$, some authors use the additional capacities $\tilde{C}_{X}=\left(\phi \frac{\partial Q}{\partial \phi}\right)_{X}$ to investigate phase transitions of black holes. 